\title{
A DISCRETE-TIME MODEL FOR REINVESTMENT RISK IN BOND MARKETS*
}

\author{
BY
}

MikKel DAHL

\begin{abstract}
In this paper we propose a discrete-time model with fixed maximum time to maturity of traded bonds. At each trading time, a bond matures and a new bond is introduced in the market, such that the number of traded bonds is constant. The entry price of the newly issued bond depends on the prices of the bonds already traded and a stochastic term independent of the existing bond prices. Hence, we obtain a bond market model for the reinvestment risk, which is present in practice, when hedging long term contracts. In order to determine optimal hedging strategies we consider the criteria of super-replication and risk-minimization.
\end{abstract}

\section{KEYWORDS}

Zero coupon bond, incomplete market, forward rates, risk-minimization, superreplication.

\section{INTRODUCTION}

In the literature, bond markets are usually assumed to include all bonds with time of maturity less than or equal to time of maturity of the considered claim. However, this is in contrast to practice, where only bonds with a limited (sufficiently short) time to maturity are traded (in a liquid market). Hence, a standard model is the correct framework for pricing and hedging so-called short term contracts, where the payoff depends on bonds with time to maturity less than or equal to the longest traded bond. However, when considering long term contracts, i.e. contracts, whose payoffs depend on bonds with longer time to maturity than the longest traded bond, the bond market does not in general include bonds, which at all times allow for a perfect hedge of the contract. Thus, in practice, an agent interested in pricing and hedging a long

* JEL classification: G10.

Mathematics Subject Classification (2000): 62P05, 91B28. 
term contract, such as a life insurance contract, where the payments may be due 50 years or more into the future, is in general not able to eliminate the reinvestment risk associated with the contract. Since the reinvestment risk is ignored in standard bond market models, they do not seem to be the right framework for pricing and hedging long term contracts. Here, we propose a model, which behaves similarly to a standard model when hedging and pricing short term contracts, and at the same time it includes reinvestment risk, when hedging long term contracts. The introduction and study of this model is the main focus of the paper. Hence, even though the problem is of practical importance for life insurance companies and other companies trading very long term contracts, qualitative rather than quantitative results are pursued.

A first idea in order to introduce reinvestment risk would be to consider a standard binomial model for the bond prices and restrict the investment strategies to bonds with a limited time to maturity only. However, this simple approach does not introduce reinvestment risk, since a long term bond can be perfectly replicated by a dynamic trading strategy, where we at all times invest in two short term bonds. Hence, we have to extend the standard model to include an additional unhedgeable stochastic term, whose uncertainty determines the reinvestment risk.

To describe the reinvestment risk, we propose a discrete-time bond market model, where the traded bonds have a fixed maximum time to maturity, $\tilde{T}$. Hence, at time 0 all bonds with time of maturity $v, v \in\{1, \ldots, \tilde{T}\}$ are traded. At any time $t$, the bond with maturity $t$ matures and a new bond with time to maturity $\tilde{T}$ is introduced in the market. Thus, after the issue of the new bond, the model is similar to the one at time 0 . At any time $t$, the entry price of the new bond is allowed to depend on all past information, current prices of bonds already traded and an additional stochastic term. In this model the class of attainable claims is time-dependent. Hence, a claim which is unattainable at time $t$ may be attainable at time $t+1$. Consider for example a claim of 1 at time $t+\tilde{T}+1$, which is unattainable at time $t$, whereas it is clearly attainable at time $t+1$, where a bond with time of maturity $t+\tilde{T}+1$ is issued. At time $t+1$ the unique arbitrage free price is equal to the price of the bond with maturity $t+$ $\tilde{T}+1$, and the replicating strategy consists of purchasing exactly one such bond. The idea of fixing the maximum time to maturity of the traded assets and introducing new assets as time passes can also be found in Neuberger (1999), who considers a market for futures on oil prices. To model the initial price of the new future, Neuberger assumes that it is a linear function of the prices of traded futures and a normally distributed error term.

To the author's knowledge, the only other papers to consider the problem of modelling the prices of newly issued bonds are Sommer (1997) and Dahl (2005), who both consider models in continuous time. In Sommer (1997), new bonds are issued continuously, whereas in Dahl (2005) new bonds are issued at fixed times only, since this is the case in practice. In order to control and quantify the reinvestment risk, both authors consider the criterion of riskminimization. 
The main contribution of the paper is the introduction of a discrete-time model dealing with the particular form of incompleteness stemming from the lack of sufficiently long bonds. Other major contributions are the derivation within the model of super-replicating and risk-minimizing strategies, which both have a nice intuitive interpretations.

The paper is organized as follows: In Section 2, a bond market model including reinvestment risk is introduced. This is done in two steps: First we describe a complete and arbitrage free standard bond market model. Then we extend the model to include reinvestment risk. Since the extended model is incomplete, there exist infinitely many equivalent martingale measures. We identify the equivalent martingale measures for the extended model and define the considered price processes, which for notational convenience are different from the bond prices. Given the considered price processes we review the relevant financial terminology. Optimal hedging strategies with respect to the criteria of super-replication and risk-minimization are determined in Section 3. Here, we also remark on the relationship between the criterion of super-replication and the maximal guarantees for which the shortfall risk can be eliminated. The paper is concluded by a numerical illustration of the mechanics of the model in Section 4. The numerical illustration includes a comparison with the pricing methodology applied to long term contracts in Danish life insurance.

\section{A BOND MARKET MODEL}

Let $\hat{T} \in \mathbb{N}$ be a fixed time horizon and $(\Omega, \mathcal{F}, P)$ a probability space with a filtration $\mathbb{F}=\left(\mathcal{F}_{t}\right)_{t \in\{0,1, \ldots, \hat{T}\}}$ satisfying the usual condition of completeness, i.e. $\mathcal{F}_{0}$ contains all $P$-null sets.

\subsection{A standard bond market model}

Prior to the introduction of the bond market model with reinvestment risk, we now describe a standard discrete-time bond market model. For a thorough description of discrete-time bond market models we refer to Jarrow (1996).

Consider a bond market where trading takes place at times $t=0,1, \ldots, \tilde{T}$, for a fixed time horizon $\tilde{T} \in \mathbb{N}, \tilde{T}<\hat{T}$. At time $t$ we assume that all zero coupon bonds with maturity $v=t, \ldots, \widetilde{T}$ are traded in the bond market. For $t \in\{0, \ldots, \widetilde{T}\}$ and $v \in\{t, \ldots, \tilde{T}\}$ we denote by $P(t, v)$ the price at time $t$ of a zero coupon bond maturing at time $v$. To avoid arbitrage we assume $P(t, v)$ is strictly positive and $P(t, t)=1$ for all $t$. For non-negative interest rates $P(t, v)$ is a decreasing function of $v$ for fixed $t$. An important quantity when modelling bond prices is the forward rate, $f_{t, v}$, contracted at time $t$ for the period $[v, v+1]$ defined by

$$
f_{t, v}=\frac{P(t, v)}{P(t, v+1)}-1, t \in\{0, \ldots, \tilde{T}-1\} \text { and } v \in\{t, \ldots, \tilde{T}-1\}
$$


or, stated differently,

$$
P(t, v)=\frac{1}{\prod_{i=t}^{v-1}\left(1+f_{t, i}\right)}, t \in\{0, \ldots, \tilde{T}-1\} \text { and } v \in\{t+1, \ldots, \tilde{T}\} .
$$

The forward rate $f_{t, v}$ can be interpreted as the riskfree interest rate contracted at time $t$ for the interval $[v, v+1]$. Now introduce the short rate process $r=\left(r_{t}\right)_{t \in\left\{0,1, \ldots, \tilde{T}_{-1}\right\}}$ given by $r_{t}=f_{t, t}$. Since (2.1) and (2.2) establish a one-to-one correspondence between forward rates and bond prices, modelling the development of the bond prices and the forward rates is equivalent. As it is standard in the literature, we model the forward rates. Let $\bar{f}_{t}=\left(r_{t}, f_{t, t+1}, \ldots, f_{t, \tilde{T}_{-1}}\right)$ denote the $(\tilde{T}-t)$-dimensional forward rate vector at time $t$. To model the development of the forward rate vector we assume

$$
\bar{f}_{t}=g_{t}\left(\bar{f}_{0}, \ldots, \bar{f}_{t-1}, \rho_{t}\right), t \in\{1, \ldots, \tilde{T}-1\},
$$

for some function $g_{t}: \mathbb{R}^{\tilde{T}} \times \mathbb{R}^{\tilde{T}-1} \times \cdots \times \mathbb{R}^{\tilde{T}-(t-1)} \times\{u, d\} \mapsto \mathbb{R}^{\tilde{T}-t}$ and an i.i.d. sequence $\rho_{1}, \ldots, \rho_{\tilde{T}-1}$ of random variables with distribution $P\left(\rho_{1}=u\right)=1-$ $P\left(\rho_{1}=d\right)=p, p \in(0,1)$. A natural restriction would be to consider strictly positive forward rates, only. In this case we would have $g_{t}: \mathbb{R}_{+}^{\tilde{T}} \times \mathbb{R}_{+}^{\tilde{T}-1} \times \cdots \times$ $\mathbb{R}_{+}^{\tilde{T}-(t-1)} \times\{u, d\} \mapsto \mathbb{R}_{+}^{\tilde{T}-t}$. We observe from (2.3) that contingent on the development of the forward rates until time $t-1$, the forward rate vector at time $t$ takes one of two possible values: $g_{t}\left(\bar{f}_{0}, \ldots, \bar{f}_{t-1}, u\right)$ or $g_{t}\left(\bar{f}_{0}, \ldots, \bar{f}_{t-1}, d\right)$. If $\bar{f}_{t}=$ $g_{t}\left(\bar{f}_{0}, \ldots, \bar{f}_{t-1}, u\right)$ we say that the forward rates have moved $u p$, and likewise, if $\bar{f}_{t}=g_{t}\left(\bar{f}_{0}, \ldots, \bar{f}_{t-1}, d\right)$ we say they have moved down. We note from (2.2) that the bond prices move in the opposite direction of the forward rates. The development of the forward rates (and bond prices) can be represented by non-recombining binomial tree, see Figure 2.1 for a visualization of the first three possible values of the forward rates.

Remark 2.1. If $g_{t}$ only depends on $\left(\bar{f}_{0}, \ldots, \bar{f}_{t-1}\right)$ through $\bar{f}_{t-1}$, then the forward rate vector is a discrete time-inhomogeneous Markov chain.

The natural filtration $\mathbb{G}=\left(\mathcal{G}_{t}\right)_{t \in\{0, \ldots, \tilde{T}\}}$ generated by the forward rates is given by

$$
\mathcal{G}_{0}=\{\emptyset, \Omega\} \text { and } \mathcal{G}_{t}=\sigma\left\{\rho_{1}, \ldots, \rho_{t \wedge(\tilde{T}-1)}\right\}, t \in\{1, \ldots, \tilde{T}\} .
$$

Introducing the notation $\xi_{t}, \xi_{t} \in \Xi_{t}=$ \{all possible sequences of $u$ 's and $d$ 's of length $t\}$ allows us to denote the generic value of for instance the forward rate vector at time $t$ by $\bar{f}_{t}^{\xi_{t}}$ and the forward rate vector at time $t+1$ given $\rho_{t+1}=d$ by $\bar{f}_{t+1}^{\xi_{t}, d}$. 


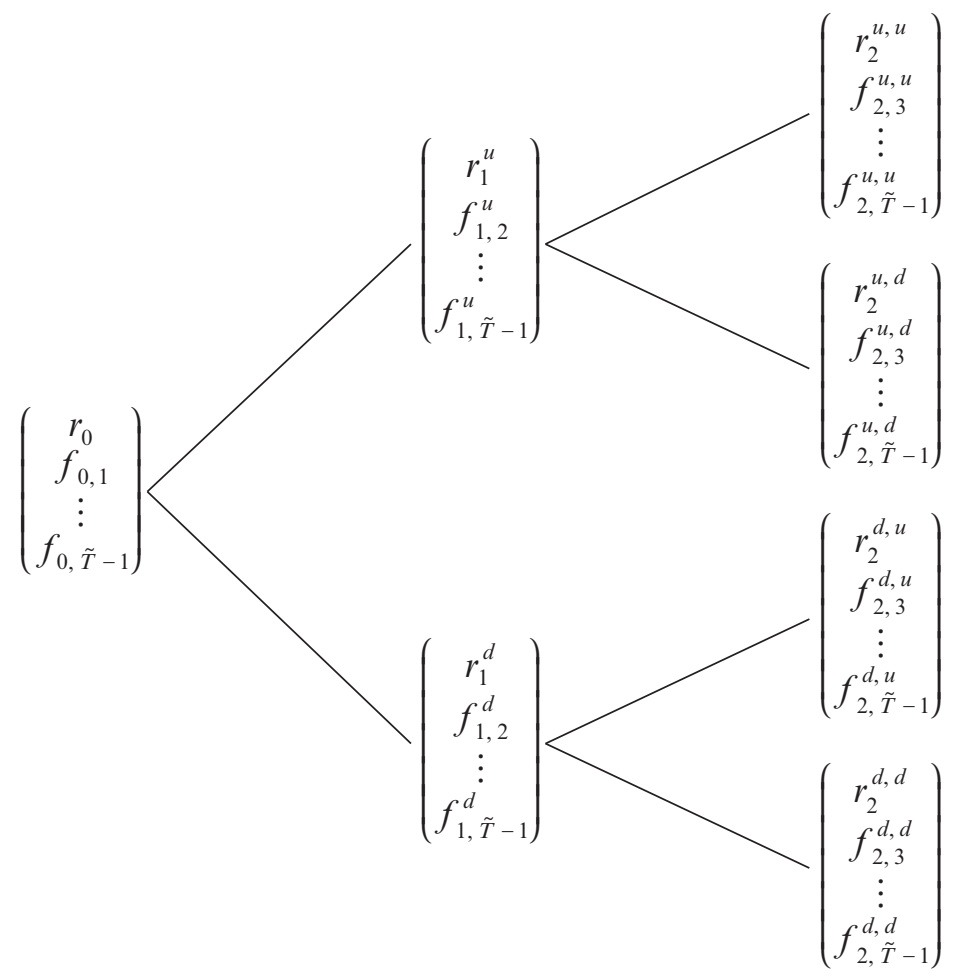

FIGURE 2.1. Development of the forward rate vector.

\subsubsection{Risk-neutral probabilities}

It is well known that the bond market model described above is arbitrage free if there exists a so-called equivalent martingale measure $Q$. Recall that an equivalent martingale measure is a probability measure equivalent to $P$, such that all discounted bond prices are martingales. The discounted bond prices are $Q$-martingales if for $t \in\{0, \ldots, \tilde{T}-1\}$ and $v \in\{t+1, \ldots, \tilde{T}\}$ it holds that

$$
P(t, v)=\frac{1}{1+r_{t}} E^{Q}\left[P(t+1, v) \mid \mathcal{G}_{t}\right] .
$$

If further the equivalent martingale measure $Q$ is unique, the model is complete; see also Section 2.3 for the definition of arbitrage and completeness. Denote by $q_{t+1}^{\xi_{t}}$ the $Q$-probability of the event $\rho_{t+1}=u$ given the present information $\xi_{t}$. Since (2.4) is trivially fulfilled for $v=t+1$, we have $\tilde{T}-(t+1)$ equations for $q_{t+1}^{\xi_{t}}, t \in\{0, \ldots, \tilde{T}-2\}$. Thus, if a solution exists, it is unique, provided there exists a $v \in\{t+2, \ldots, \tilde{T}\}$, such that $P_{\xi_{t}, d}(t+1, v) \neq P^{\xi_{t}, u}(t+1, v)$. For $t \in$ $\{0, \ldots, \tilde{T}-2\}$, solving (2.4) gives the following expressions for $q_{t+1}^{\xi_{t}}$ : 


$$
q_{t+1}^{\xi_{t}}=\frac{P^{\xi_{t}, d}(t+1, v)-\left(1+r_{t}^{\xi_{t}}\right) P^{\xi_{t}}(t, v)}{P^{\xi_{t}, d}(t+1, v)-P^{\xi_{t}, u}(t+1, v)}, v \in\{t+2, \ldots, \tilde{T}\} .
$$

Here, we have used the notation $r_{t}^{\xi_{t}}$ and $P^{\xi_{t}}(t, v)$ to denote explicitly the dependence on the past. Since (2.5) must hold simultaneous for all the considered values of $v$ a necessary condition for the existence of an equivalent martingale measure is that the right hand side of (2.5) is independent of $v$. From (2.5) we observe that the $Q$-probability of $\rho_{t+1}=u$ depends on $\xi_{t}$ and hence in general differs for different outcomes of $\left(\rho_{1}, \ldots, \rho_{t}\right)$. Furthermore, $(2.5)$ gives that the $Q$-probability of an upward movement is small (large) if the difference $P^{\xi_{t}, d}(t+1, v)-\left(1+r_{t}^{\xi_{t}}\right) P^{\xi_{t}}(t, v)$ is small (large) compared to the difference $P^{\xi_{t}, d}(t+1, v)-P^{\xi_{t}, u}(t+1, v)$. The measure $\mathrm{Q}$ given by (2.5) for all $t$ ensures that all discounted bond price processes are $Q$-martingales. If further $q_{t+1}^{\xi_{t}} \in(0,1)$ for all $t$ and $\xi_{t}$, then $P$ and $Q$ are equivalent measures, such that $Q$ indeed is an equivalent martingale measure. From (2.5) we get that $Q$ and $P$ are equivalent if for all $t \in\{0, \ldots, \tilde{T}-2\}$ and $\xi_{t}$ it holds that

$$
P^{\xi_{t}, u}(t+1, v)<\left(1+r_{t}^{\xi_{t}}\right) P^{\xi_{t}}(t, v)<P^{\xi_{t}, d}(t+1, v), v \in\{t+2, \ldots, \tilde{T}\} .
$$

Here, we have used that $P^{\xi_{t}, u}(t+1, v)<P^{\xi_{t}, d}(t+1, v)$, since an upward movement of the forward rates corresponds to a downward move of the bond prices. Using (2.2) one can alternatively express (2.5) and (2.6) in terms of the forward rates. Condition (2.6) can be interpreted as follows: No bond with time to maturity larger than one must dominate or be dominated by the 1-period bond. If this was the case we could make arbitrage by trading in the particular bond and the 1-period bond.

\subsection{A bond market model with reinvestment risk}

Now, we extend the standard model in Section 2.1 to include reinvestment risk. The idea is as follows: Assume that at any time $t$ only bonds with time to maturity less than or equal to $\tilde{T}$ are traded, and the development of the bond prices from time $t$ to $t+1$ can be described by a binomial model. Hence, at time $t$ the one period development of the traded bonds is the same as in the standard model introduced in Section 2.1. At time $t+1$ the bond with maturity $t+1$ matures and a new bond with time to maturity $\widetilde{T}$ is issued, such that after the introduction of the new bond the model considered is similar to the one at time $t$. To model the reinvestment risk we assume that conditional on the past and the prices at time $t+1$ of the bonds traded at time $t$, the entry price at time $t+1$ of the new bond with time to maturity $\tilde{T}$ can take two different values.

Consider a bond market where trading takes place at times $t=0,1, \ldots, \hat{T}$. In this bond market not all zero coupon bonds with maturity less than or equal 
to $\hat{T}$ are traded at all times $t=0,1, \ldots, \hat{T}$. Instead we fix the maximum time to maturity, $\tilde{T}$, for bonds traded in the market. Hence, the zero coupon bond prices $P(t, v)$ are defined for $t \in\{0, \ldots, \hat{T}\}$ and $v \in\{t, \ldots,(t+\tilde{T}) \wedge \hat{T}\}$. In addition to $\hat{T}$ and $\tilde{T}$ we introduce the fixed time horizon $T \in \mathbb{N}$, which is the time of maturity of the considered contract. Figure 2.2 shows the possible orderings of $T$, $\tilde{T}$ and $\hat{T}$.

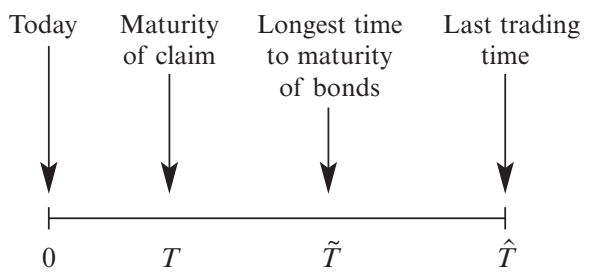

(a)

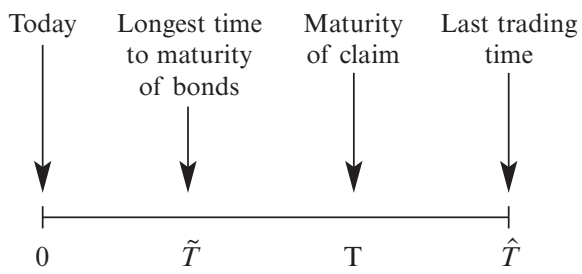

(b)

FIgURE 2.2. Possible orderings of $T, \tilde{T}$ and $\hat{T}$. In case (a) all fixed claims with maturity $T$ are attainable. In (b) they are unattainable.

Without loss of generality we assume that $\hat{T}=T+\tilde{T}$, such that the bond market at all times $t, t \in\{0, \ldots, T\}$, includes the $\tilde{T}$ bonds with time of maturity $v$, $v \in\{t+1, \ldots, t+\tilde{T}\}$. From (2.1) we observe that the forward rates are defined for $t \in\{0, \ldots, \hat{T}-1\}$ and $v \in\{t, \ldots,(t+\tilde{T}-1) \wedge(\hat{T}-1)\}$, so the forward rate vector at time $t$ (which we still denote by $\left.\bar{f}_{t}\right)$ is given by $\bar{f}_{t}=\left(r_{t}, f_{t, t+1}, \ldots, f_{t,(t+\tilde{T}-1) \wedge(\hat{T}-1)}\right)$. Define the $((u-t+1) \wedge \tilde{T})$-dimensional vector $\bar{f}_{t, u}$ of forward rates defined at time $t$ with time of maturity less than or equal to $u$. Now assume that

$$
\bar{f}_{t,(t+\tilde{T}-2) \wedge(\hat{T}-1)}=z_{t}\left(\bar{f}_{0}, \ldots, \bar{f}_{t-1}, \rho_{t}\right),
$$

where $z_{t}: \mathbb{R}^{\tilde{T}} \times \mathbb{R}^{\tilde{T} \wedge(\hat{T}-1)} \times \ldots \times \mathbb{R}^{\tilde{T} \wedge(\hat{T}-(t-1))} \times\{u, d\} \mapsto \mathbb{R}$, and $\rho_{1}, \ldots, \rho_{\hat{T}-1}$, similarly to Section 2.1, is an i.i.d. sequence of random variables with distribution $P\left(\rho_{1}=u\right)=1-P\left(\rho_{1}=d\right)=p, p \in(0,1)$. The filtration $\mathbb{G}=\left(G_{t}\right)_{t \in\{0, \ldots, \hat{T}\}}$ is now given by

$$
\mathcal{G}_{0}=\{\emptyset, \Omega\} \text { and } \mathcal{G}_{t}=\sigma\left\{\rho_{1}, \ldots, \rho_{t \wedge(\hat{T}-1)}\right\}, \quad t \in\{1, \ldots, \hat{T}\} .
$$

At time $t$ the maturities of the forward rates given by (2.7) are those where a forward rate with the same maturity is defined at time $t-1$. Hence, the forward rates at time $t$ given by (2.7) determine the bond prices at time $t$ for bonds with time of maturity $v, v \in\{t+1, \ldots,(t+\tilde{T}-1) \wedge \hat{T}\}$, which are the bonds traded at time $t-1$ (when disregarding the bond maturing at time $t$ ). Thus, the uncertainty associated with the development of the forward rates (bond prices) from time $t-1$ to $t$ is described by $\rho_{t}$. However, the uncertainty associated with the price of the new bond with time to maturity $\tilde{T}$ introduced in the market at time $t, t \in\{1, \ldots, T\}$, cannot be described entirely by $\rho_{t}$; it depends on an additional 
source of risk. In order to model this additional uncertainty we assume that at time $t, t \in\{1, \ldots, T\}$, the $(\tilde{T}-1)$-period forward rate, $f_{t, t+\tilde{T}-1}$, is given by

$$
f_{t, t+\tilde{T}-1}=c_{t}\left(\bar{f}_{0}, \ldots, \bar{f}_{t-1}, \bar{f}_{t, t+\tilde{T}-2}, \varepsilon_{t}\right)
$$

for some function $c_{t}:\left(\mathbb{R}^{\tilde{T}}\right)^{t} \times \mathbb{R}^{\tilde{T}-1} \times\{h, \ell\} \mapsto \mathbb{R}$ and an i.d.d. sequence $\varepsilon_{1}, \ldots, \varepsilon_{T}$ of random variables independent of $\left(\rho_{t}\right)_{t \in\left\{1, \ldots, \hat{T}_{-1}\right\}}$. The distribution of $\varepsilon_{1}$ is given by $P\left(\varepsilon_{1}=h\right)=1-P\left(\varepsilon_{1}=\ell\right)=\check{p}, \check{p} \in(0,1)$. Hence, for $t \in\{1, \ldots, T\}$ it holds that given the past forward rates and the $(\tilde{T}-1)$-dimensional forward rate vector $\tilde{f}_{t, t+\tilde{T}_{-2}}=\left(r_{t}, f_{t, t+1}, \ldots, f_{t, t+\tilde{T}-2}\right)$ at time $t$, the $(\tilde{T}-1)$-period forward rate, $f_{t, t+\tilde{T}-1}$, can attain two different values: $c_{t}\left(\bar{f}_{0}, \ldots, \bar{f}_{t-1}, \bar{f}_{t, t}+\tilde{T}_{-2}, h\right)$ and $c_{t}\left(\bar{f}_{0}, \ldots\right.$, $\left.\bar{f}_{t-1}, f_{t, t+\tilde{T}-2}, \ell\right)$. We refer to these values as the high and low value, respectively. Analogously to $\xi_{t}$ we now introduce $\lambda_{t}, \lambda_{t} \in \Lambda_{t}=$ \{all possible sequences of $h$ 's and $\ell$ 's of length $t\}$ for all $t=0, \ldots, T$. Thus, $\lambda_{t}$ keeps track of whether the past values of the $(\tilde{T}-1)$-period forward rate has attained the high or the low value. Hence, $\xi_{t}$ and $\lambda_{t \wedge T}$ determine the development of the entire forward rate vector until time $t, t \in\{1, \ldots, \hat{T}-1\}$, such that we can denote the generic value of the forward rate vector at time $t$ by $\bar{f}_{t} \bar{\xi}_{t} \lambda_{t \wedge}$. Now introduce the filtration $\mathbb{H}=$ $\left(\mathcal{H}_{t}\right)_{t \in\{0, \ldots, \hat{T}\}}$ by

$$
\mathcal{H}_{0}=\{\emptyset, \Omega\} \text { and } \mathcal{H}_{t}=\sigma\left\{\varepsilon_{1}, \ldots, \varepsilon_{t \wedge T}\right\}, t \in\{1, \ldots, \hat{T}\} .
$$

We now assume the filtration $\mathbb{F}=\left(\mathcal{F}_{t}\right)_{t \in\{0,1, \ldots, \hat{T}\}}$ introduced earlier is given by

$$
\mathcal{F}_{t}=\mathcal{G}_{t} \vee \mathcal{H}_{t}
$$

Hence, $\mathbb{F}$ is the filtration for the extended bond market. We note that it is sufficient to consider the state space for $\omega$ given by $\Omega=\{u, d\}^{\hat{T}-1} \times\{h, l\}^{T}$ and the $\sigma$-algebra $\mathcal{F}=\mathcal{F}_{\hat{T}}=\mathcal{F}_{\hat{T}-1}$.

At time $t, t \in\{T+1, \ldots, \hat{T}\}$ the development of the bond market is essentially identical to the binomial model in Section 2.1, whereas the model is nonstandard at time $t, t \in\{1, \ldots, T\}$. Here, we have that contingent on $\xi_{t-1}$ and $\lambda_{t-1}$ there are four possible forward rate vectors at time $t$ and hence $4^{t}$ possible states at time $t$. Thus, for $t \leq T$ the development of the forward rate vector can be represented using a non-recombining quadrinomial tree, see Figure 2.3 for a visualization of the forward rates with $\tilde{T}=2$.

From (2.7) we observe that the forward rate at time $t$ with maturity $\tau, \tau \in$ $\{t, \ldots,(t+\tilde{T}-2) \wedge(\hat{T}-1)\}$, is allowed to depend on all past forward rates, such that the $(\tilde{T}-1)$-period forward rate at time $t-1$ may influence the entire forward rate vector at time $t$. Hence, the forward rate $f_{t, \tau}, \tau \in\{t, \ldots,(t+\tilde{T}-2) \wedge(\hat{T}-1)\}$, is $\left(\mathcal{F}_{t-1} \vee \mathcal{G}_{t}\right)$-measurable, which in turn gives that $P(t, \tau)$ also is $\left(\mathcal{F}_{t-1} \vee \mathcal{G}_{t}\right)$-measurable for $\tau \in\{t+1, \ldots,(t+\tilde{T}-1) \wedge \hat{T}\}$. For an illustration of the dependence of the forward rates on the $\rho$ 's and $\varepsilon$ 's we again refer to Figure 2.3, where the dependence is shown explicitly. 
Note that if we contingent on the outcome of the vector $\left(\varepsilon_{1}, \ldots, \varepsilon_{T}\right)$, the development can be described by a binomial model, and hence the conditional model is complete, such that in the conditional model zero coupon bonds with all maturities have unique prices (even before they are traded). Hence, in the conditional model we, at all times, have a forward rate vector for all maturities. However, in the unconditional model the future values of $\varepsilon_{t+1}, \ldots, \varepsilon_{T}$ are unknown at time $t$, such that it is uncertain which of the conditional forward rate vectors in retrospect will turn out to have been "the correct one", when $\varepsilon_{t+1}, \ldots, \varepsilon_{T}$ have been observed at time $T$. Thus, the reinvestment risk can be interpreted as the uncertainty associated with which of the conditional forward rate vectors in retrospect has turned out to have been "the correct one". This in turn gives that the magnitude of the reinvestment risk is related to how much the conditional forward rate vectors differ.

Remark 2.2. For reasons of simplicity both the development of the known forward rate curve and the reinvestment risk are described by a simple 1-factor model. This allows us to more clearly illustrate the idea and explain the mechanics of the model. In practice a more complex model would probably lead to a more realistic description of the two types of risk. Moreover we note that the complexity of (2.8) with regards to the dependence of present and past forward rates is irrelevant for the magnitude of the reinvestment risk faced by a company. This risk is entirely described by the dependence in (2.8) on the $\varepsilon$ 's.

Example 2.3. Consider the case where $\tilde{T}=2$ and $T=3$. Hence, the time to maturity of the longest bond in the market is 2 and the time of maturity of the considered claim is 3 . The development of the forward rate vector can be visualized by Figure 2.3. Here, the superscripts denote the dependence of the forward rates on the outcome of the variables $\rho$ and $\varepsilon$. As an example the notation $r_{2}^{u u, \ell}$ denotes the short rate in period 2 if $\rho_{1}=u, \rho_{2}=u$ and $\varepsilon_{1}=\ell$. We end the example by noting that all examples in this paper are one continuing example.

\subsubsection{Risk-neutral probabilities}

We now aim at determining the equivalent martingale measures in the extended model. Here, the uncertainty is generated by $\left(\rho_{t}\right)_{t \in\left\{1, \ldots, \hat{T}_{-1}\right\}}$ and $\left(\varepsilon_{t}\right)_{t \in\{1, \ldots, T\}}$, such that the measure $Q$ is uniquely determined by $\left(q_{t}^{\left.\xi_{t-1}, \lambda_{(t-1) \wedge T}\right)} t \in\{1, \ldots, \hat{T}-1\}\right.$ and

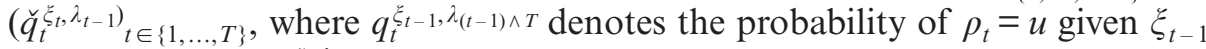
and $\lambda_{(t-1) \wedge T}$, and $\breve{q}_{t}^{\xi_{t}}, \lambda_{t-1}$ denotes the probability of $\varepsilon_{t}=h$ given $\xi_{t}$ and $\lambda_{t-1}$. Recall that for $t \in\{0, \ldots, \hat{T}-1\}$ and $v \in\{t+1, \ldots,(t+\tilde{T}) \wedge \hat{T}\}$, a necessary condition for $Q$ to be an equivalent martingale measure is

$$
P(t, v)=\frac{1}{1+r_{t}} E^{Q}\left[P(t+1, v) \mid \mathcal{F}_{t}\right]
$$




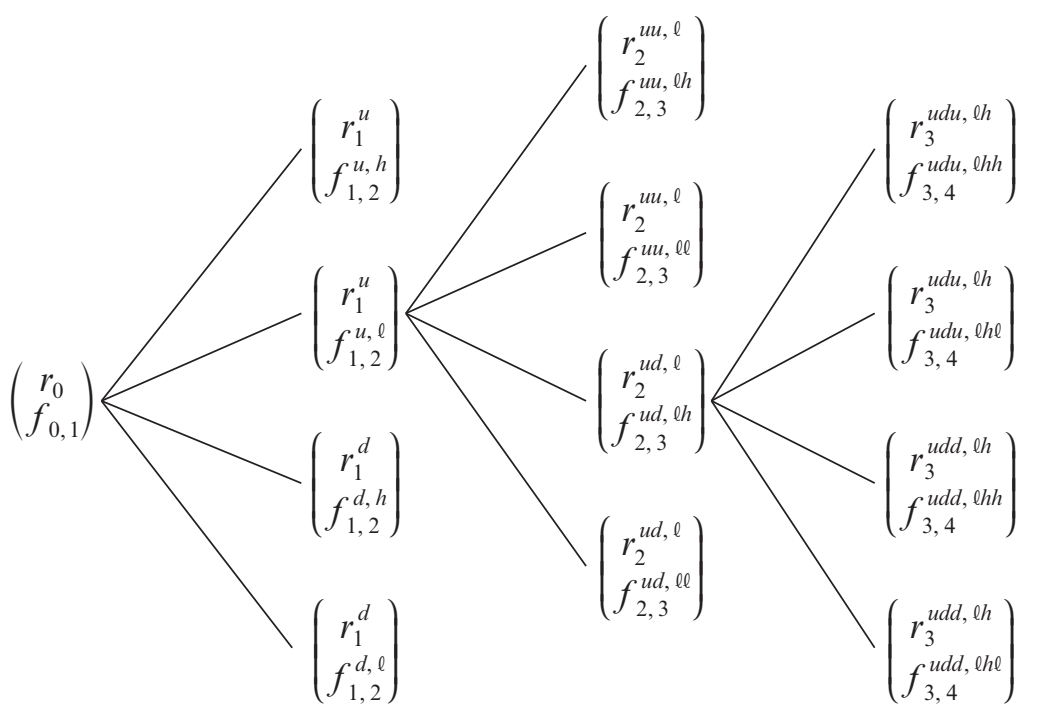

FIGURE 2.3: Development of the forward rates in the extended model with $\tilde{T}=2$.

such that the discounted bond prices are martingales. Now note that (2.9) is trivially fulfilled if $v=t+1$. Since $P(t+1, v)$ in (2.9) is $\mathcal{F}_{t} \vee \mathcal{G}_{t+1}$-measurable, i.e. independent of $\varepsilon_{t+1}$, then (2.9) yields $(\tilde{T}-2) \wedge(\hat{T}-t-1)$ equations for $q_{t+1}^{\xi_{t}, \lambda_{t \wedge}}$. Hence, if there exists an equivalent martingale measure, then $q_{t+1}^{\xi_{t}, \lambda_{1} \wedge T}$ is unique for all $t \in\{0, \ldots, \hat{T}-2\}$, provided there for each $t$ exists a $v \in\{t+2, \ldots,(t+\tilde{T}) \wedge \tilde{T}\}$, such that $P^{\xi_{t}, \lambda_{t}, d}(t+1, v) \neq P^{\xi_{t}, \lambda_{t}, u}(t+1, v)$. For $t \in\{0, \ldots, T-1\}$ no information regarding $\check{q}_{t+1}^{\xi_{t+1}, \lambda_{t}}$ can be derived from (2.9), so any $Q$ for which $q_{t+1}^{\xi_{t}, \lambda_{t \wedge T}}$ fulfills (2.9) for all $t \in\{0, \ldots, \hat{T}-2\}$ ensures that the discounted bond prices are martingales. If further both $q_{t+1}^{\xi_{t}, \lambda_{t} \wedge T}$ and $\breve{q}_{t+1}^{\xi_{t+1}, \lambda_{t}}$ lie in the interval $(0,1)$, then $Q$ is an equivalent martingale measure. If $\breve{q}_{t+1}^{\xi_{t+1}, \lambda_{t}}=\breve{p}$, we say the market is risk-neutral with respect to reinvestment risk. This measure is known as the minimal martingale measure for the extended model, i.e. the equivalent martingale measure which "disturbs the structure of the model as little as possible", see Schweizer (1995). Here, we restrict ourselves to $Q$ 's given by $\check{q}_{t+1}^{\xi_{t+1}, \lambda_{t}}=\breve{q}_{t+1}^{\lambda_{t}}$, such that under $Q$ it holds that the distribution of the $\varepsilon$ 's is independent of the realization of the $\rho$ 's. Henceforth we consider a fixed, but arbitrary, equivalent martingale measure $Q$.

Remark 2.4. Note that for $t \in\{0, \ldots, \hat{T}-1\}$ and $v \in\{t+1, \ldots,(t+\tilde{T}) \wedge \hat{T}\}$ repeated use of (2.9) gives the equation

$$
P(t, v)=\frac{1}{1+r_{t}} E^{Q}\left[\frac{1}{1+r_{t+1}} E^{Q}\left[P(t+2, v) \mid \mathcal{F}_{t+1}\right] \mid \mathcal{F}_{t}\right] .
$$


Hence, since $P(t+2, v)$ is $\left(\mathcal{F}_{t+1} \vee \mathcal{G}_{t+2}\right)$-measurable it seems as if (2.10) gives an equation from which to determine $\breve{q}_{t+1}^{\xi_{t+1}, \lambda_{t}}$ for $t \in\{0, \ldots, T-1\}$. However, this is not the case, since the $\left(\mathcal{F}_{t} \vee \mathcal{G}_{t+1}\right)$-measurability of $P(t+1, v)$ ensures that $E^{Q}\left[P(t+2, v) \mid \mathcal{F}_{t+1}\right]$ is independent of $\varepsilon_{t+1}$.

\subsubsection{Model considerations}

Consider the case where we for fixed $t$ model the forward rate $f_{t, u}, u \in\{t, \ldots,(t+$ $\tilde{T}-2) \wedge(\hat{T}-1)\}$ by

$$
f_{t, u}=z_{t, u}\left(\bar{f}_{0, u}, \ldots, \bar{f}_{t-1, u}, \rho_{t}\right), \quad t \in\{1, \ldots, \hat{T}-1\},
$$

where $z_{t, u}: \mathbb{R}^{(u+1) \wedge \tilde{T}} \times \cdots \times \mathbb{R}^{(u-t+2) \wedge \tilde{T}} \times\{u, d\} \mapsto \mathbb{R}$. Hence, the development of the forward rates given by (2.11) is a special case of (2.7), where we have restricted the possible dependence on the past forward rates. Here, the forward rate at time $t$ with maturity $\tau, \tau \in\{t, \ldots,(t+\tilde{T}-2) \wedge(\hat{T}-1)\}$, is allowed to depend on the past forward rates with maturity less than or equal to $\tau$ only. This in turn gives that $f_{t, \tau}$ is independent of $\varepsilon_{v}$ for $v>((\tau-\tilde{T}+1) \vee 0)$. With this restriction we have that the price at time $t$ of a bond with time of maturity $\tau \in\{t, \ldots,(t+\tilde{T}-1) \wedge \hat{T}\}$ is $\left(\mathcal{F}_{\tau-\tilde{T}} \vee \mathcal{G}_{t}\right)$-measurable. Here, and throughout the paper, we adopt the convention that $\mathcal{F}_{-\tau}=\mathcal{F}_{0}$ for $\tau \in \mathbb{N}$. Within this model we have that once a bond is introduced in the market, the development of the price process is entirely described by the outcome of the $\rho$ 's. Hence, at time $t$ we essentially are in the complete and arbitrage free model from Section 2.1 when considering the filtration $\mathbb{G}$ and the time horizon $(t+\tilde{T}-1) \wedge \hat{T}$.

\subsection{Discrete-time trading}

Since the bonds traded in the bond market depend on the time considered, it is inconvenient to define trading strategies in terms of the bonds. Hence, we define $\tilde{T}$ new price processes $\left(S^{k}\right)_{k=1, \ldots, \tilde{T}}$, which are defined for all $t=0,1, \ldots, T$, by $S_{0}^{k}=1$ and

$$
S_{t}^{k}=\frac{P(t, t-1+k)}{P(t-1, t-1+k)} S_{t-1}^{k}=\prod_{i=0}^{t-1} \frac{P(i+1, i+k)}{P(i, i+k)}, t \in\{1, \ldots, T\} .
$$

We note that until time $T$ these price processes include exactly the same information as the original bond prices. The price process $S^{k}$ is generated by investing 1 unit at time 0 in bonds with time to maturity $k$ and at times $t=1, \ldots, T$ selling the bonds with time to maturity $k-1$ purchased at time $t-1$ and reinvesting the money in bonds with time to maturity $k$. Hence, for $k \in\{1, \ldots, \tilde{T}\}$ 
the price process $S^{k}$ is the value process generated by a roll-over strategy in bonds with time to maturity $k$. Such a value process is usually referred to as a rolling-horizon bond, see Rutkowski (1999). Recall that in a discrete-time model, the 1-period bond is equal to a savings account, so the price process $S^{1}$ corresponds to investing in a savings account with a locally riskfree interest rate. Note that given $\mathcal{F}_{t-1}$, the future value of the price process vector at time $t$, $\left(S_{t}^{k}\right)_{k \in\left\{1, \ldots, \tilde{T}_{\}}\right.}$, depends on $\rho_{t}$ only, such that it is sufficient for hedging purposes to consider any two of the rolling-horizon bonds defined by (2.12). Here, we consider the savings account, henceforth denoted $B$, and $S^{\tilde{T}}$, henceforth simply denoted $S$. We shall refer to the asset with price process $S$ as the risky asset. Note that the measurability conditions on the bond prices give that $B_{t}$ and $S_{t}$, respectively, are $\left(\mathcal{F}_{t-2} \vee \mathcal{G}_{t-1}\right)$ - and $\left(\mathcal{F}_{t-1} \vee \mathcal{G}_{t}\right)$-measurable.

A trading strategy with respect to $(B, S)$ is an adapted two-dimensional process $\varphi=(\vartheta, \eta)$. Hence, $\vartheta_{t}$ and $\eta_{t}$ are $\mathcal{F}_{t}$-measurable for all $t$. The pair $\varphi_{t}=\left(\vartheta_{t}, \eta_{t}\right)$ is interpreted as the portfolio established at time $t$ and held until time $t+1$. Here, $\vartheta_{t}$ denotes the number of risky assets held in the portfolio, and $\eta_{t}$ is the discounted deposit in the savings account. The value process associated with the trading strategy $\varphi$ is denoted $\mathcal{V}(\varphi)$. Here, $\mathcal{V}_{t}(\varphi)$, which is the value at time $t$ of holding the portfolio $\left(\vartheta_{t}, \eta_{t}\right)$, is given by

$$
\mathcal{V}_{t}(\varphi)=\vartheta_{t} S_{t}+\eta_{t} B_{t} .
$$

With the definition of $\vartheta$ and $\eta$ above the value process is seen to be the value after any in- or outflow of capital at time $t$. A trading strategy is called selffinancing if for all $t$

$$
\mathcal{V}_{t}(\varphi)=\mathcal{V}_{0}(\varphi)+\sum_{u=0}^{t-1} \vartheta_{u} \Delta S_{u+1}+\sum_{u=0}^{t-1} \eta_{u} \Delta B_{u+1}
$$

where we have introduced the notation exemplified by $\Delta S_{u}=S_{u}-S_{u-1}$. Thus, the value at time $t$ of a self-financing strategy is the initial value added interest and investment gains from trading in the bond market. Hence, withdrawals or deposits are not allowed at intermediate times $t=1, \ldots, T-1$. A self-financing strategy is a so-called arbitrage if $\mathcal{V}_{0}(\varphi)=0$ and $\mathcal{V}_{T}(\varphi) \geq 0 P$-a.s. with $P\left(\mathcal{V}_{T}(\varphi)>0\right)>0$. A contingent claim (or a derivative) with maturity $T$ is an $\mathcal{F}_{T}$-measurable random variable $H$. A contingent claim is called attainable if there exists a selffinancing strategy $\varphi$ such that $\mathcal{V}_{T}(\varphi)=H P$-a.s. An attainable claim can thus be replicated perfectly by investing $\mathcal{V}_{0}(\varphi)$ at time 0 and adjusting the portfolio at times $t=1, \ldots, T-1$, according to the self-financing strategy $\varphi$. Hence, at any time $t$, there is no difference between holding the claim $H$ and the portfolio $\varphi_{t}$. In this sense, the claim $H$ is redundant in the market and from the assumption of no arbitrage it follows that the price of $H$ at time $t$ must be $\mathcal{V}_{t}(\varphi)$. Thus, the initial investment $\mathcal{V}_{0}(\varphi)$ is the unique arbitrage free price of $H$. Note that if $\varphi$ is a self-financing portfolio replicating the contingent claim $H$, then (2.14) gives the following representation for $H$ : 


$$
H=\mathcal{V}_{T}(\varphi)=\mathcal{V}_{0}(\varphi)+\sum_{u=0}^{T-1} \vartheta_{u} \Delta S_{u+1}+\sum_{u=0}^{T-1} \eta_{u} \Delta B_{u+1}
$$

If all contingent claims are attainable, the model is called complete and otherwise it is called incomplete. Throughout the paper, we denote by $S^{*}, \mathcal{V}^{*}(\varphi)$ and $H^{*}$ the discounted price process of the risky asset, the discounted value process and the discounted claim, respectively.

Remark 2.5. The definition of trading strategies in discrete time is not uniform in the literature. Harrison and Kreps (1979), Jarrow (1996) and Musiela and Rutkowski (1997) define trading strategies as adapted processes, whereas Harrison and Pliska (1981) and Björk (2004) consider predictable processes. The different measurability conditions lead to one significant difference, namely, whether the value process defined by (2.13) denotes the value before or after a possible withdrawal or deposit. A third possibility is the definition in Föllmer and Schweizer (1988). They consider a predictable process $\vartheta$ and an adapted process $\eta$. Hence, the portfolio at time $t$ is given by the number of risky assets held in the portfolio from time $t-1$ to $t$ and the discounted deposit in the savings account after a possible withdrawal or deposit. Since they define the value process after a possible withdrawal or deposit their value process coincides with the value process in the present paper. Hence, we have the following connection between our definition of trading strategies and the Föllmer-Schweizer definition:

$$
\begin{aligned}
& \vartheta_{t}=\vartheta_{t+1}^{F S} \\
& \eta_{t}=\eta_{t}^{F S}+\left(\vartheta_{t}^{F S}-\vartheta_{t+1}^{F S}\right) S_{t}^{*} .
\end{aligned}
$$

Here, $\left(\vartheta_{t}^{F S}, \eta_{t}^{F S}\right)$ denotes the portfolio at time $t$ using the Föllmer-Schweizer definition.

Example 2.6. If $\tilde{T}=2$ and $T=3$ then the price processes for the savings account and the risky asset are given by

$$
\begin{aligned}
& B_{0}=1, B_{t}=\prod_{i=0}^{t-1}\left(1+r_{i}\right), t \in\{1,2,3\} \text { and } \\
& S_{0}=1, S_{t}=\prod_{i=0}^{t-1} \frac{P(i+1, i+2)}{P(i, i+2)}, t \in\{1,2,3\},
\end{aligned}
$$

respectively. Here, one easily observes that, as noted above, $B_{t}$ is $\left(\mathcal{F}_{t-2} \vee G_{t-1}\right)$ measurable and $S_{t}$ is $\left(\mathcal{F}_{t-1} \vee \mathcal{G}_{t}\right)$-measurable. 


\section{Hedging STRATEGIES}

Consider a company interested in hedging the claim $H$ with maturity $T$. If $H$ only depends on bonds with time of maturity at time $\tilde{T}$ or earlier, it has a unique arbitrage free price and can be replicated perfectly leaving the company without any risk. However, if $H$ depends on bonds maturing after time $\tilde{T}$, then $H$ does in general not have a perfect replicating strategy, and hence in general it does not have a unique arbitrage free price. For unattainable claims we determine the optimal hedging strategies for the criteria of super-replication and risk-minimization.

\subsection{Super-replication}

A strategy $\varphi$ is called super-replicating for the claim $H$ with maturity $T$ if the value process is of the form

$$
\mathcal{V}_{t}(\varphi)=\mathcal{V}_{0}(\varphi)+\sum_{u=0}^{t-1} \vartheta_{u} \Delta S_{u+1}+\sum_{u=0}^{t-1} \eta_{u} \Delta B_{u+1}-U_{t}
$$

where $U$ is a non-decreasing process, and the terminal value of the value process satisfies $\mathcal{V}_{T}(\varphi) \geq H P$-a.s. Here, the process $U$ is the accumulated outflow of capital when using the strategy $\varphi$. Thus, when following a super-replicating strategy no inflow of capital is needed in addition to the initial investment in order to guarantee that at time of maturity, the value of the portfolio is at least as large as the considered claim. Hence, following a super-replicating strategy allows the hedger to eliminate the risk of falling short of the claim. The smallest initial value needed at time $t$ to construct a super-replicating strategy is referred to as the super-replicating price at time $t$, henceforth denoted $\hat{\pi}_{t}(H)$. Hence, the superreplicating price at time $t$ is the smallest initial investment at time $t$ allowing the company to hedge the considered claim without any risk of falling short. For more details on super-replication see El Karoui and Quenez (1995) and Föllmer and Schied (2002). Now define the super-replicating price process as the process of the super-replicating prices, i.e. the value of the super-replicating price process at time $t$ is exactly the super-replicating price at time $t$. At any time $t$ the optimal super-replicating strategy is defined as the super-replicating strategy corresponding to the super-replicating price process. Prior to the general result for the super-replicating price process and optimal super-replicating strategy for a claim $H$ with maturity $T$, we first consider super-replication in a 1-period model.

\section{Lemma 3.1.}

At time $t, t \in\{0, \ldots, T-1\}$, the optimal super-replicating strategy, $\hat{\varphi}_{t}=\left(\hat{\vartheta}_{t}, \hat{\eta}_{t}\right)$, for a claim $H$ with time of maturity $t+1$ is given by

$$
\hat{\vartheta}_{t}=\frac{\widehat{H}(d)-\widehat{H}(u)}{S_{t+1}^{d}-S_{t+1}^{u}} \text { and } \hat{\eta}_{t}=\frac{\widehat{H}(u) S_{t+1}^{d}-\widehat{H}(d) S_{t+1}^{u}}{B_{t+1}\left(S_{t+1}^{d}-S_{t+1}^{u}\right)},
$$


where

$$
\widehat{H}\left(\rho_{t+1}\right)=\max \left(H\left(\rho_{t+1}, h\right), H\left(\rho_{t+1}, \ell\right)\right), \rho_{t+1} \in\{u, d\} .
$$

The super-replicating price is

$$
\hat{\pi}_{t}(H)=\frac{1}{1+r_{t}}\left(q_{t+1} \widehat{H}(u)+\left(1-q_{t+1}\right) \widehat{H}(d)\right) .
$$

Proof of Lemma 3.1: Consider an agent holding the portfolio $\left(\vartheta_{t}, \eta_{t}\right)$ at time $t$. Before any adjustments at time $t+1$ the value of the portfolio can take one of two values: $\vartheta_{t} S_{t+1}^{u}+\eta_{t} B_{t+1}$ or $\vartheta_{t} S_{t+1}^{d}+\eta_{t} B_{t+1}$. Hence, the value of the portfolio is the same in the states $(u, h)$ and $(u, \ell)$, as well as in $(d, h)$ and $(d, \ell)$. Thus, for $\left(\vartheta_{t}, \eta_{t}\right)$ to be super-replicating it must hold that

$$
\begin{aligned}
& \vartheta_{t} S_{t+1}^{u}+\eta_{t} B_{t+1} \geq \max (H(u, h), H(u, \ell)), \\
& \vartheta_{t} S_{t+1}^{d}+\eta_{t} B_{t+1} \geq \max (H(d, h), H(d, \ell)),
\end{aligned}
$$

where the $H(i, j)$ denotes the payoff from $H$ if $\rho_{t+1}=i$ and $\varepsilon_{t+1}=j$, where $i \in$ $\{u, d\}$ and $j \in\{h, \ell\}$. Define the contingent claim $\widehat{H}$ with payoff

$$
\widehat{H}\left(\rho_{t+1}\right)=\max \left(H\left(\rho_{t+1}, h\right), H\left(\rho_{t+1}, \ell\right)\right), \quad \rho_{t+1} \in\{u, d\},
$$

and note that the strategy

$$
\vartheta_{t}=\frac{\widehat{H}(d)-\widehat{H}(u)}{S_{t+1}^{d}-S_{t+1}^{u}} \text { and } \eta_{t}=\frac{\widehat{H}(u) S_{t+1}^{d}-\widehat{H}(d) S_{t+1}^{u}}{B_{t+1}\left(S_{t+1}^{d}-S_{t+1}^{u}\right)}
$$

replicates $\widehat{H}$. A no arbitrage argument now gives that the replicating strategy and the unique arbitrage free price for $\widehat{H}$ is the optimal super-replicating strategy and super-replicating price, respectively.

Lemma 3.1 has the following interpretation: The dependence of $H$ on $\varepsilon_{t+1}$ is unhedgeable. Hence, for each outcome of $\rho_{t+1}$ we assume the outcome of $\varepsilon_{t+1}$ which leads to the highest value of $H$ and replicate this claim. The replicating strategy and the unique arbitrage free price of this "worst scenario" claim are then equal to the optimal super-replicating strategy and super-replicating price, respectively.

Remark 3.2. The main result in Aliprantis, Polyrakis and Tourky (2002) states that in a 1-period model the optimal super-replicating strategy shall be found among the replicating strategies in the complete sub-models arising from eliminating states of the world. Hence, Lemma 3.1 can be seen as a special case, where the optimal super-replicating strategy is easily identifiable. 


\section{Theorem 3.3}

Consider a claim $H$ with time of maturity $T$. For $t \in\{0, \ldots, T-1\}$ the portfolio, $\hat{\varphi}_{t}=\left(\hat{\vartheta}_{t}, \hat{\eta}_{t}\right)$ held in the optimal super-replicating strategy is given by

$$
\begin{aligned}
\hat{\vartheta}_{t}^{\xi_{t}, \lambda_{t}} & =\frac{\hat{\pi}_{t+1}^{\xi_{t}, d, \lambda_{t}}(H)-\hat{\pi}_{t+1}^{\xi_{t}, u, \lambda_{t}}(H)}{S_{t+1}^{\xi_{t}, d, \lambda_{t}}-S_{t+1}^{\xi_{t}, u, \lambda_{t}}} \text { and } \\
\hat{\eta}_{t}^{\xi_{t}, \lambda_{t}} & =\frac{\hat{\pi}_{t+1}^{\xi_{t}, u, \lambda_{t}}(H) S_{t+1}^{\xi_{t}, d, \lambda_{t}}-\hat{\pi}_{t+1}^{\xi_{t}, d, \lambda_{t}}(H) S_{t+1}^{\xi_{t}, u, \lambda_{t}}}{B_{t+1}^{\xi_{t}, \lambda_{t-1}}\left(S_{t+1}^{\xi_{t}, d, \lambda_{t}}-S_{t+1}^{\xi_{t}, u, \lambda_{t}}\right)},
\end{aligned}
$$

where

$$
\hat{\pi}_{t+1}^{\xi_{t}, \rho_{t+1}, \lambda_{t}}(H)=\max \left(\hat{\pi}_{t+1}^{\xi_{t}, \rho_{t+1}, \lambda_{t}, h}(H), \hat{\pi}_{t+1}^{\xi_{t}, \rho_{t+1}, \lambda_{t}, \ell}(H)\right), \rho_{t+1} \in\{u, d\} .
$$

Starting with the terminal value

$$
\hat{\pi}_{T}^{\xi_{T}, \lambda_{T}}(H)=H,
$$

the super-replicating price process at time $t, t \in\{0, \ldots, T-1\}$, is given by the following recursive formula

$$
\hat{\pi}_{t}^{\xi_{t}, \lambda_{t}}(H)=\frac{1}{1+r_{t}^{\xi_{t}, \lambda_{(t-1) \vee 0}}}\left(q_{t+1}^{\xi_{t}, \lambda_{t}} \hat{\pi}_{t+1}^{\xi_{t}, u, \lambda_{t}}(H)+\left(1-q_{t+1}^{\xi_{t}, \lambda_{t}}\right) \hat{\pi}_{t+1}^{\xi_{t}, d, \lambda_{t}}(H)\right) .
$$

Proof of Theorem 3.3: First note that at time $T$ the super-replicating price is trivial and equal to $H$. At time $t, t \in\{0, \ldots, T-1\}$ we may consider the superreplicating price at time $t+1, \hat{\pi}_{t+1}(H)$, as the payoff from at contingent claim with maturity $t+1$. Thus, Lemma 3.1 gives the super-replicating price and optimal super-replicating strategy at time $t$ in terms of the super-replicating price at time $t+1$.

Note that we in Theorem 3.3 explicitly denote the dependence on the past through $\xi_{t}$ and $\lambda_{t}$ in order to emphasize the dependence of the optimal superreplicating strategy and super-replicating price process on the past.

For sufficiently nice claims, such as fixed claims, the following corollary allows for an easy calculation of the super-replicating price process and the optimal super-replicating strategy.

\section{Corollary 3.4}

If for each $t, t \in\{0, \ldots, T-1\}$, it holds, for fixed $k_{t+1} \in\{h, l\}$ that

$$
\hat{\pi}_{t+1}^{\xi_{t}, \rho_{t+1}, \lambda_{t}}(H)=\hat{\pi}_{t+1}^{\xi_{t}, \rho_{t+1}, \lambda_{t}, k_{t+1}}(H)
$$


for all $\xi_{t}, \rho_{t+1}$ and $\lambda_{t}$, then the super-replicating price and optimal super-replicating strategy at time $\tau$ are, respectively, the unique arbitrage free price and the replicating strategy in the conditional model given $\left(\varepsilon_{\tau+1}, \ldots, \varepsilon_{T}\right)=\left(k_{\tau+1}, \ldots, k_{T}\right)$.

Denote by $\widehat{U}$ the process $U$ from (3.1) associated with the optimal super-replicating strategy. Hence, $\widehat{U}$ denotes the accumulated outflow of capital, when using the optimal super-replicating strategy. Combining (3.1) and Theorem 3.3 gives the following explicit expression for the change in $\widehat{U}$ at time $t$

$$
\Delta \widehat{U}_{t}^{\xi_{t-1}, \rho_{t}, \lambda_{t-1}, \varepsilon_{t}}=\hat{\pi}_{t}^{\xi_{t-1}, \rho_{t}, \lambda_{t-1}}(H)-\hat{\pi}_{t}^{\xi_{t-1}, \rho_{t}, \lambda_{t-1}, \varepsilon_{t}}(H) .
$$

Investigating (3.2) we observe that the withdrawal is the difference between the value at time $t$ of the optimal super-replicating portfolio purchased at time $t-1$ and the super-replicating price at time $t$. Hence, when using the optimal super-replicating strategy the withdrawal from the portfolio at time $t$ depends on the outcome of the two random variables observed at time $t, \rho_{t}$ and $\varepsilon_{t}$. Given (3.2), one easily derives the conditional expectation under $P$ of $\Delta \widehat{U}_{t}^{\xi_{t-1}, \rho_{t}, \lambda_{t-1}, \varepsilon_{t}}$ given $\mathcal{F}_{t-1}$, namely,

$$
\begin{aligned}
& E^{P}\left[\Delta \widehat{U}_{t}^{\xi_{t-1}, \rho_{t}, \lambda_{t-1}, \varepsilon_{t}}(H) \mid \mathcal{F}_{t-1}\right] \\
& =E^{P}\left[\hat{\pi}_{t}^{\xi_{t-1}, \rho_{t}, \lambda_{t-1}}(H)-\hat{\pi}_{t}^{\xi_{t-1}, \rho_{t}, \lambda_{t-1}, \varepsilon_{t}}(H) \mid \mathcal{F}_{t-1}\right] \\
& =p\left(\hat{\pi}_{t}^{\xi_{t-1}, u, \lambda_{t-1}}(H)-\left(\check{p} \hat{\pi}_{t}^{\xi_{t-1}, u, \lambda_{t-1}, h}(H)+(1-\check{p}) \hat{\pi}_{t}^{\xi_{t-1}, u, \lambda_{t-1}, \ell}(H)\right)\right) \\
& +(1-p)\left(\hat{\pi}_{t}^{\xi_{t-1}, d, \lambda_{t-1}}(H)-\left(\check{p} \hat{\pi}_{t}^{\xi_{t-1}, d, \lambda_{t-1}, h}(H)+(1-\check{p}) \hat{\pi}_{t}^{\xi_{t-1}, d, \lambda_{t-1}, \ell}(H)\right)\right) .
\end{aligned}
$$

Thus, the expected withdrawal from the optimal super-replicating portfolio is the probability of an upward jump multiplied by the expected withdrawal contingent on an upward jump added the probability of a downward jump multiplied by the expected withdrawal in this case.

Example 3.5. Let $\tilde{T}=2, T=3$ and $H=1$. Since $H$ is attainable at time 1 and $\hat{\pi}_{1}^{\rho_{1}}=\hat{\pi}_{1}^{\rho_{1}, \ell}$, we have from Corollary 3.4 that the super-replicating price and the super-replicating strategy at time 0 corresponds to using the conditional forward rate vector given $\varepsilon_{1}=\ell$. Hence, we obtain the following super-replicating price process, expressed in terms of bond prices:

$$
\begin{aligned}
& \hat{\pi}_{3}^{\xi_{3}, \lambda_{3}}(1)=1, \\
& \hat{\pi}_{2}^{\xi_{2}, \lambda_{2}}(1)=\frac{1}{1+r_{2}^{\xi_{2}, \lambda_{1}}}\left(q_{3}^{\xi_{2}, \lambda_{2}}+\left(1-q_{3}^{\xi_{2}, \lambda_{2}}\right)\right)=\frac{1}{1+r_{2}^{\xi_{2}, \lambda_{1}}}=P^{\xi_{2}, \lambda_{1}}(2,3),
\end{aligned}
$$




$$
\begin{aligned}
\hat{\pi}_{1}^{\xi_{1}, \lambda_{1}}(1) & =\frac{1}{1+r_{1}^{\xi_{1}}}\left(q_{2}^{\xi_{1}, \lambda_{1}} P^{\xi_{1}, u, \lambda_{1}}(2,3)+\left(1-q_{2}^{\xi_{1}, \lambda_{1}}\right) P^{\xi_{1}, d, \lambda_{1}}(2,3)\right)=P^{\xi_{1}, \lambda_{1}}(1,3), \\
\hat{\pi}_{0}(1) & =\frac{1}{1+r_{0}}\left(q_{1} P^{u, \ell}(1,3)+\left(1-q_{1}\right) P^{d, \ell}(1,3)\right) .
\end{aligned}
$$

The optimal super-replicating strategy is given by

$$
\begin{aligned}
& \left(\hat{\vartheta}_{0}, \hat{\eta}_{0}\right)=\left(\frac{P(0,2)\left(P^{d, \ell}(1,3)-P^{u, \ell}(1,3)\right)}{P^{d}(1,2)-P^{u}(1,2)}, \frac{P^{d}(1,2) P^{u, \ell}(1,3)-P^{u}(1,2) P^{d, \ell}(1,3)}{\left(1+r_{0}\right)\left(P^{d}(1,2)-P^{u}(1,2)\right)}\right) \\
& \left(\hat{\vartheta}_{1}^{\xi_{1}, \lambda_{1}}, \hat{\eta}_{1}^{\xi_{1}, \lambda_{1}}\right)=\left(\frac{P(0,2) P^{\xi_{1}, \lambda_{1}}(1,3)}{P^{\xi_{1}}(1,2)}, 0\right) \text { and }\left(\hat{\vartheta}_{2}^{\xi_{2}, \lambda_{2}}, \hat{\eta}_{2}^{\xi_{2}, \lambda_{2}}\right)=\left(0, \frac{1}{B_{3}^{\xi_{2}, \lambda_{1}}}\right) .
\end{aligned}
$$

Remark 3.6. Note that the worst case scenario is strongly dependent on the model and period length. In this case where the unhedgeable reinvestment risk is modelled by a binomial model the difference between the expected and the worst possible outcome increases by approximately a factor of root two as the period length is halved.

\subsubsection{Relation to guarantees}

Apart from the nice property of allowing the hedger to eliminate the shortfall risk the super-replicating price process relates to the maximal possible guarantees for which the risk of falling short can be eliminated. Here, we consider two types of guarantees: Maturity guarantees and periodic interest rate guarantees. Given a deposit at time $t$ the maturity guarantee is the minimal possible payoff at time $T$, whereas the periodic interest guarantee is the minimum interest earned on the deposit in each period until time $T$. We shall refer to the maximal guarantees for which the short fall risk can be eliminated as the maximal riskfree maturity guarantee and maximal riskfree periodic interest rate guarantee.

\section{Proposition 3.7}

Given an initial deposit of 1 at time t, the maximal riskfree maturity guarantee, $G_{t}^{T}$, at time $T$ is given by

$$
G_{t}^{T}=\frac{1}{\hat{\pi}_{t}(1)} .
$$

At time t the maximal riskfree periodic interest rate guarantee with maturity $T$, $g_{t}^{T}$, is 


$$
g_{t}^{T}=\left(\frac{1}{\hat{\pi}_{t}(1)}\right)^{\frac{1}{T-t}}-1
$$

Proof of Proposition 3.7: At time $t$ the super-replicating price of 1 unit at time $T$ is given by $\hat{\pi}_{t}(1)$. Hence, by investing 1 at time $t$ we may purchase $1 / \hat{\pi}_{t}(1)$ units of the super-replicating strategy. This guarantees a payoff at time $T$ of at least $1 / \hat{\pi}_{t}(1)$. Hence, since the super-replicating price per definition is the lowest initial deposit for which a certain payoff is guaranteed, the maximal riskfree maturity guarantee at time $T$ is given by (3.3). Now, the maximal riskfree periodic interest rate guarantee is the constant short rate which gives a payoff of $G_{t}^{T}$ at time $T$, when depositing 1 unit at time $t$. Hence, $g_{t}^{T}$ is the unique solution greater than -1 to

$$
\left(1+g_{t}^{T}\right)^{T-t}=G_{t}^{T}
$$

Inserting (3.3) and isolating $g_{t}^{T}$ now gives (3.4).

Proposition 3.7 is of importance to for instance life insurance companies, since it gives the maximal guarantees, which the companies should promise the insured at initiation of the contract.

\subsection{Risk-minimizing strategies}

As an alternative to the hedging criterion of super-replication we now consider risk-minimization. Here, we give a brief review of risk-minimization and determine risk-minimizing strategies in the presence of reinvestment risk. We note that since we define trading strategies differently than Föllmer and Schweizer (1988) and Møller (2001) our results cannot be compared directly to their results.

\subsubsection{A brief review of risk-minimization}

In this section we review the criterion of risk-minimization introduced in discrete time by Föllmer and Schweizer (1988). The presentation is based on Møller (2001).

The idea of risk-minimization is closely related to the introduction of the cost process defined by

$$
C_{t}(\varphi)=\mathcal{V}_{t}^{*}(\varphi)-\sum_{u=0}^{t-1} \vartheta_{u} \Delta S_{u+1}^{*}
$$

Thus, the cost process is the discounted value of the portfolio reduced by discounted trading gains. The cost process measures the accumulated discounted 
cost of an agent following the strategy $\varphi$. Comparing (2.15) and (3.5) we note that the cost process is constant $P$-a.s. if and only if the strategy $\varphi$ is selffinancing. To measure the risk associated with the strategy $\varphi$ we introduce the risk process defined by

$$
R_{t}(\varphi)=E^{Q}\left[\left(C_{T}(\varphi)-C_{t}(\varphi)\right)^{2} \mid \mathcal{F}_{t}\right] .
$$

Hence, the risk process is the conditional expected value of the squared future costs associated with the strategy $\varphi$. A trading strategy $\varphi$ is called risk-minimizing for the contingent claim $H$ if for all $t \in\{0, \ldots, T\}$ it minimizes $R_{t}(\varphi)$ over all trading strategies with $\mathcal{V}_{T}^{*}(\varphi)=H^{*}$.

The construction of risk-minimizing strategies is based on the $Q$-martingale

$$
V_{t}^{*}=E^{Q}\left[H^{*} \mid \mathcal{F}_{t}\right]
$$

known as the intrinsic value process. Using the so-called Kunita-Watanabe decomposition for martingales, $V^{*}$ can be uniquely decomposed as

$$
V_{t}^{*}=V_{0}^{*}+\sum_{u=1}^{t} \vartheta_{u}^{H} \Delta S_{u}^{*}+L_{t}^{H},
$$

where $\vartheta^{H}$ is predictable, and $L^{H}$ is a zero-mean $Q$-martingale orthogonal to $S^{*}$, i.e. $S^{*} L^{H}$ is a $Q$-martingale as well. For more details on the Kunita-Watanabe decomposition we refer to Föllmer and Schied (2002). Shifting the index in (3.7) and defining the adapted process $\widetilde{\vartheta}^{H}$ by $\widetilde{\vartheta}_{u}^{H}=\vartheta_{u+1}^{H}$ we have the following decomposition

$$
V_{t}^{*}=V_{0}^{*}+\sum_{u=0}^{t-1} \tilde{\vartheta}_{u}^{H} \Delta S_{u+1}^{*}+L_{t}^{H}
$$

Comparing (2.15) and (3.8) we observe that $H$ is attainable if and only if $L_{T}^{H}=0$ $Q$-a.s. Using (2.16) and (2.17) we obtain the following theorem, due to Föllmer and Schweizer (1988), which relates the Kunita-Watanabe decomposition to the risk-minimizing strategy.

\section{Theorem 3.8}

There exists a unique risk-minimizing strategy, $\varphi^{*}$, with $\mathcal{V}_{T}^{*}(\varphi)=H^{*}$ given by

$$
\left(\vartheta_{t}^{*}, \eta_{t}^{*}\right)=\left(\vartheta_{t+1}^{H}, V_{t}^{*}-\vartheta_{t+1}^{H} S_{t}^{*}\right) .
$$

Inserting (3.9) in (3.5) and using the Kunita-Watanabe decomposition from (3.7) we obtain the following expression for the cost process associated with the risk-minimizing strategy:

$$
C_{t}\left(\varphi^{*}\right)=V_{t}^{*}-\sum_{u=0}^{t-1} \vartheta_{u+1}^{H} \Delta S_{u+1}^{*}=V_{0}^{*}+L_{t}^{H} .
$$


Combining (3.10) and (3.6) now gives the following expression for the so-called intrinsic risk process, which is the risk process associated with the risk-minimizing strategy:

$$
R_{t}\left(\varphi^{*}\right)=E^{Q}\left[\left(L_{t}^{H}-L_{t}^{H}\right)^{2} \mid \mathcal{F}_{t}\right]
$$

Note that when determining the risk-minimizing strategy we consider all admissible strategies. This is in contrast to many other quadratic hedging criteria, such as mean-variance hedging, where only self-financing strategies are allowed. From (3.10) we observe that risk-minimizing strategies are not self-financing for non-attainable claims. However, they are mean-self-financing, i.e. the corresponding cost processes are $Q$-martingales.

Since (3.6) involves an expectation with respect to $Q$, the risk-minimizing strategy depends on the chosen equivalent martingale measure. Furthermore we observe from (3.6) that the criterion of risk-minimization, like other quadratic hedging criteria, penalizes gains and losses equally. This is of course disadvantageous, however, when using a criterion penalizing only losses, explicit results are hard to obtain; see the discussion in Møller (2001) and references therein.

In general, the risk-minimizing strategy is given by the (predictable) $Q$ expectation of the replicating strategy given the unhedgeable uncertainty, see Schweizer (1994) for a proof in a continuous-time setup. A particular simple risk-minimizing strategy is obtained in Møller (2001), since he considers an unhedgeable risk, which is stochastically independent of the financial market. As we shall see below in Theorem 3.10, the expression for the risk-minimizing strategy is slightly more complicated in the present model than in Møller (2001), since the unhedgeable risk is in the financial market.

\subsubsection{Risk-minimizing strategies in the presence of reinvestment risk}

We now turn to the derivation of risk-minimizing strategies in the present model including reinvestment risk. In order to determine the Kunita-Watanabe decomposition of $V^{*}$ we introduce the $Q$-martingales

$$
M_{t}^{\lambda_{T}}=E^{Q}\left[1_{\left(\left(\varepsilon_{1}, \ldots, \varepsilon_{T}\right)=\lambda_{T}\right)} \mid \mathcal{F}_{t}\right]=E^{Q}\left[1_{\left(\left(\varepsilon_{1}, \ldots, \varepsilon_{T}\right)=\lambda_{T}\right)} \mid \mathcal{H}_{t}\right]
$$

for all $\lambda_{T} \in \Lambda_{T}$. Here, we have used that under $Q$ the distribution of the $\varepsilon$ 's is independent of the filtration $\mathbb{G}$. Using the quantities defined in (3.12), we get the following expression for $V_{t}^{*}$ :

$$
V_{t}^{*}=E^{Q}\left[H^{*} \mid \mathcal{F}_{t}\right]=E^{Q}\left[E^{Q}\left[H^{*} \mid \mathcal{F}_{t} \vee \mathcal{H}_{T}\right] \mid \mathcal{F}_{t}\right]=\sum_{\lambda_{T} \in \Lambda_{T}} M_{t}^{\lambda_{T}} \pi_{t}^{\lambda_{T}, *}(H)
$$

where $\pi_{t}^{\lambda_{T}, *}(H)$ is the unique discounted arbitrage free price for $H$ given $\left(\varepsilon_{1}, \ldots, \varepsilon_{T}\right)=\lambda_{T}$. Using $(3.13)$ we obtain the following expression for the development of $V^{*}$ from time $t-1$ to $t$, 


$$
\begin{aligned}
& \Delta V_{t}^{\prime}=V_{t}^{\prime}-V_{t-1}^{\prime} \\
& =\sum_{\lambda_{T} \in \Lambda_{T}} M_{t}^{\lambda_{T}} \pi_{t}^{\lambda_{T}, *}(H)-\sum_{\lambda_{T} \in \Lambda_{T}} M_{t-1}^{\lambda_{T}} \pi_{t-1}^{\lambda_{T}}{ }^{*}(H) \\
& =\sum_{\lambda_{T} \in \Lambda_{T}}\left(M_{t}^{\lambda_{T}} \pi_{t}^{\lambda_{T},{ }^{*}}(H)-M_{-1}^{\lambda_{T}} \pi_{t-1}^{\lambda_{T}}{ }^{*}(H)\right) \\
& =\sum_{\lambda_{T} \in \Lambda_{T}}\left(\left(M_{t}^{\lambda_{T}}-M_{t-1}^{\lambda_{T}}\right) \pi_{t}^{\lambda_{T},{ }^{*}}(H)+M_{t-1}^{\lambda_{T}}\left(\pi_{t}^{\lambda_{T},{ }^{*}}(H)-\pi_{t-1}^{\lambda_{T},{ }^{*}}(H)\right)\right) \\
& =\sum_{\lambda_{T} \in \Lambda_{T}}\left(\pi_{t}^{\lambda_{T},{ }^{*}}(H) \Delta M_{t}^{\lambda_{T}}+M_{t-1}^{\lambda_{T}} \Delta \pi_{t}^{\lambda_{T},{ }^{*}}(H)\right) \\
& =\sum_{\lambda_{T} \in \Lambda_{T}}\left(\pi_{t}^{\lambda_{T}{ }^{*}}(H) \Delta M_{t}^{\lambda^{2}}+M_{t-1}^{\lambda_{T}} \vartheta_{t-1}^{\lambda_{T}} \Delta S_{t}^{*}\right),
\end{aligned}
$$

where $\vartheta_{T}^{\lambda}$ is the number of risky assets in the replicating strategy in the complete model given $\left(\varepsilon_{1}, \ldots, \varepsilon_{T}\right)=\lambda_{T}$. Hence, we have the following decomposition of $V^{*}$ :

$$
V_{t}^{*}=V_{0}^{*}+\sum_{u=1}^{t}\left(\sum_{\lambda_{T} \in \Lambda_{T}} M_{u-1}^{\lambda_{T}} \vartheta_{u-1}^{\lambda_{T}}\right) \Delta S_{u}^{*}+\sum_{u=1}^{t} \sum_{\lambda_{T} \in \Lambda_{T}} \pi_{u}^{\lambda_{T},{ }^{*}}(H) \Delta M_{u}^{\lambda^{T}} .
$$

In order to show that (3.14) actually is the Kunita-Watanabe decomposition of $V^{*}$, we first note that $\sum_{\lambda_{T} \in \Lambda_{T}} M_{t-1}^{\lambda_{T}} \vartheta_{t-1}^{\lambda_{T}}$ is $\mathcal{F}_{t-1}$-measurable, such that the process $\vartheta^{H}$ defined by

$$
\vartheta_{t}^{H}=\sum_{\lambda_{T} \in \Lambda_{T}} M_{t-1}^{\lambda_{T}} \vartheta_{t-1}^{\lambda_{T}}
$$

is predictable. Now define the process $L^{H}$ by

$$
L_{t}^{H}=\sum_{u=1}^{t} \sum_{\lambda_{T} \in \Lambda_{T}} \pi_{u}^{\lambda_{T}{ }^{*}}(H) \Delta M_{u}^{\lambda_{T}} .
$$

Using the law of iterated expectations we see that

$$
\begin{aligned}
E^{Q}\left[\Delta L_{t}^{H} \mid \mathcal{F}_{t-1}\right] & =E^{Q}\left[\sum_{\lambda_{T} \in \Lambda_{T}} \pi_{t}^{\lambda_{T},{ }^{*}}(H) \Delta M_{t}^{\lambda_{T}} \mid \mathcal{F}_{t-1}\right] \\
& =\sum_{\lambda_{T} \in \Lambda_{T}} E^{Q}\left[\pi_{t}^{\lambda_{T},{ }^{*}}(H) \Delta M_{t}^{\lambda_{T}} \mid \mathcal{F}_{t-1}\right] \\
& =\sum_{\lambda_{T} \in \Lambda_{T}} E^{Q}\left[\pi_{t}^{\lambda_{T},{ }^{*}}(H) E^{Q}\left[\Delta M_{t}^{\lambda_{T}} \mid \mathcal{G}_{t} \vee \mathcal{H}_{t-1}\right] \mid \mathcal{F}_{t-1}\right] \\
& =0,
\end{aligned}
$$


since $M^{\lambda T}$ is a martingale stochastically independent of the filtration $\mathbb{G}$. Hence, $L^{H}$ is a $Q$-martingale. To show that that $L^{H} S^{*}$ is a $Q$-martingale we first observe that

$$
\Delta\left(L^{H} S^{*}\right)_{t}=L_{t}^{H} S_{t}^{*}-L_{t-1}^{H} S_{t-1}^{*}=L_{t-1}^{H} \Delta S_{t}^{*}+S_{t-1}^{*} \Delta L_{t}^{H}+\Delta L_{t}^{H} \Delta S_{t}^{*} .
$$

Thus, since $L^{H}$ and $S^{*}$ are $Q$-martingales, it is sufficient to show that

$$
\begin{aligned}
E^{Q}\left[\Delta L_{t} \Delta S_{t}^{*} \mid \mathcal{F}_{t-1}\right] & =E^{Q}\left[\sum_{\lambda_{T} \in \Lambda_{T}} \pi_{t}^{\lambda_{T},}{ }^{*}(H) \Delta M_{t}^{\lambda_{T}} \Delta S_{t}^{*} \mid \mathcal{F}_{t-1}\right] \\
& =\sum_{\lambda_{T} \in \Lambda_{T}} E^{Q}\left[\pi_{t}^{\lambda_{T},{ }^{*}}(H) \Delta M_{t}^{\lambda_{T} T} \Delta S_{t}^{*} \mid \mathcal{F}_{t-1}\right] \\
& =\sum_{\lambda_{T} \in \Lambda_{T}} E^{Q}\left[\pi_{t}^{\lambda_{T},{ }^{*}}(H) \Delta S_{t}^{*} E^{Q}\left[\Delta M_{t}^{\lambda_{T}} \mid \mathcal{G}_{t} \vee \mathcal{H}_{t-1}\right] \mid \mathcal{F}_{t-1}\right] \\
& =0 .
\end{aligned}
$$

Hence, we have proved the following.

\section{Lemma 3.9}

For a claim $H$ with time of maturity $T$ the Kunita-Watanabe decomposition is given by

$$
V_{t}^{*}=V_{0}^{*}+\sum_{u=1}^{t}\left(\sum_{\lambda_{T} \in \Lambda_{T}} M_{u-1}^{\lambda_{T}} \vartheta_{u-1}^{\lambda_{T}}\right) \Delta S_{u}^{*}+\sum_{u=1}^{t} \sum_{\lambda_{T} \in \Lambda_{T}} \pi_{u}^{\lambda_{T},{ }^{*}}(H) \Delta M_{u}^{\lambda_{T}} .
$$

Combining Lemma 3.9, Theorem 3.8 and the expression for the intrinsic risk process in (3.11) we obtain the following theorem regarding the risk-minimizing strategy and the intrinsic risk process.

\section{Theorem 3.10}

The risk-minimizing strategy, $\varphi^{*}$, for $H$ is given by

$$
\left(\vartheta_{t}^{*}, \eta_{t}^{*}\right)=\left(\sum_{\lambda_{T} \in \Lambda_{T}} M_{t}^{\lambda_{T}} \vartheta_{t}^{\lambda_{T}}, \sum_{\lambda_{T} \in \Lambda_{T}} M_{t}^{\lambda_{T}} \pi_{t}^{\lambda_{T},{ }^{*}}(H)-\left(\sum_{\lambda_{T} \in \Lambda_{T}} M_{t}^{\lambda_{T}} \vartheta_{t}^{\lambda_{T}}\right) S_{t}^{*}\right) .
$$

The intrinsic risk process is given by

$$
R_{t}\left(\varphi^{*}\right)=E^{Q}\left[\left(\sum_{u=t+1}^{T} \sum_{\lambda_{T} \in \Lambda_{T}} \pi_{u}^{\lambda_{T},{ }^{*}}(H) \Delta M_{u}^{\lambda_{T}}\right)^{2} \mid \mathcal{F}_{t}\right] .
$$

Thus, the number of risky assets held in the risk-minimizing strategy at time $t$ is the average under $Q$ of the replicating strategies for $H$ in the conditional 
models given the outcome of $\left(\varepsilon_{1}, \ldots, \varepsilon_{T}\right)$. The deposit in the savings account is adjusted each period according to the realization of the unhedgeable variables, such that the discounted value process is equal to the intrinsic value process.

Inserting (3.15) in (3.10) gives the following expression for the cost process associated with $\varphi^{*}$ :

$$
C_{t}\left(\varphi^{*}\right)=V_{0}^{*}+\sum_{u=1}^{t} \sum_{\lambda_{T} \in \Lambda_{T}} \pi_{u}^{\lambda_{T},{ }^{*}}(H) \Delta M_{u}^{\lambda^{T}} .
$$

From (3.16) we see that the change in the cost process at time $t$ for an agent following the risk-minimizing strategy depends on the change in the $Q$-martingales $M^{\lambda_{T}}$ associated with the outcome of $\varepsilon_{t}$. If the claim is attainable at some time $t$ prior to $T$, then the cost process is constant $P$-a.s. from time $t$, and hence, the intrinsic risk process is zero from time $t$.

Example 3.11. Let $\tilde{T}=2, T=3$ and $H=1$. For the fixed $Q$-measure given by $\breve{q}^{\lambda_{t-1}}=\breve{q} \in(0,1)$ we now obtain the risk-minimizing strategy from Theorem 3.10. At time 0 the risk-minimizing strategy is given by

$$
\begin{aligned}
\vartheta_{0}^{*}= & P(0,2)\left(\breve{q} \frac{P^{d, h}(1,3)-P^{u, h}(1,3)}{P^{d}(1,2)-P^{u}(1,2)}+(1-\breve{q}) \frac{P^{d, \ell}(1,3)-P^{u, \ell}(1,3)}{P^{d}(1,2)-P^{u}(1,2)}\right), \\
\eta_{0}^{*}= & \breve{q} \frac{P^{d}(1,2) P^{u, h}(1,3)-P^{u}(1,2) P^{d, h}(1,3)}{\left(1+r_{0}\right)\left(P^{d}(1,2)-P^{u}(1,2)\right)} \\
& +(1-\breve{q}) \frac{P^{d}(1,2) P^{u, \ell}(1,3)-P^{u}(1,2) P^{d, \ell}(1,3)}{\left(1+r_{0}\right)\left(P^{d}(1,2)-P^{u}(1,2)\right)},
\end{aligned}
$$

whereas it at time 1 and 2 is given by

$\left(\vartheta_{1}^{\xi_{1}, \lambda_{1}, *}, \eta_{1}^{\xi_{1}, \lambda_{1},{ }^{*}}\right)=\left(\frac{P(0,2) P^{\xi_{1}, \lambda_{1}}(1,3)}{P^{\xi_{1}}(1,2)}, 0\right)$ and $\left(\vartheta_{2}^{\xi_{2}, \lambda_{2},{ }^{*}}, \eta_{2}^{\xi_{2}, \lambda_{2}, *}\right)=\left(0, \frac{1}{B_{3}^{\xi_{2}, \lambda_{1}}}\right)$.

We note that since the claim is attainable from time 1, the risk-minimizing strategy and super-replicating strategies coincide at times 1 and 2. Moreover, since the strategies coincide so do the super-replicating price and the value of the portfolio held in the risk-minimizing strategy.

\section{A NumericAl illustration}

As already noted in the introduction the purpose of this illustration is to provide a simple example illustrating the mechanism of the model and hedging 
techniques. A full-scale (continuous-time) example illustrating the effect of reinvestment risk on prices and risk measures will be treated in a separate paper. Now we aim at providing some numbers for the continuing example considered in Sections 2 and 3. Hence, $\tilde{T}=2, T=3$ and $H=1$. Now assume that given the initial forward rate vector $\left(r_{0}, f_{0,1}\right)$ the forward rates at time $t, t \in\{1,2,3\}$, are given by

$$
\begin{aligned}
r_{t}^{\xi_{t}, \lambda_{t-1}} & =r_{0} \prod_{i=1}^{t}\left(a_{1} 1_{\left(\rho_{i}=u\right)}+a_{2} 1_{\left(\rho_{i}=d\right)}\right) \prod_{i=1}^{t-1}\left(a_{3} 1_{\left(\varepsilon_{i}=h\right)}+a_{4} 1_{\left(\varepsilon_{i}=\ell\right)}\right), \\
f_{t, t+1}^{\xi_{t}, \lambda_{t}} & =r_{t}^{\xi_{t}, \lambda_{t-1}}\left(b_{1} 1_{\left(\varepsilon_{t}=h\right)}+b_{2} 1_{\left(\varepsilon_{t}=\ell\right)}\right),
\end{aligned}
$$

where $a_{1}, \ldots, a_{4}, b_{1}, b_{2}$ are positive constants, and $\prod_{i=1}^{u}$ is interpreted as 1 if $u=0$. The constants $a_{1}$ and $a_{2}$ describe the movement of the forward rate vector due to the outcome of the $\rho$ 's, whereas $a_{3}$ and $a_{4}$ describe the dependence of the forward rate vector on past values of the $\varepsilon$ 's, and finally $b_{1}$ and $b_{2}$ describe the unhedgeable uncertainty associated with the newly issued bonds. In this simple model the dependence on $\xi_{t}$ is given by the number of $u$ 's and not by the ordering of the $u$ 's. Hence, the number of states at time 2 is reduced from 16 to 12. However, this is still a large number of states compared to the 4 in a binomial model ( 3 if the binomial model is recombining). In contrast to an additive structure, the multiplicative structure above ensures that the forward rates are strictly positive. Now let the initial forward rate curve and the constants be given by $r_{0}=0.03, f_{0,1}=0.031, a_{1}=1.25, a_{2}=0.8, a_{3}=1.01, a_{4}=0.99, b_{1}=1.0325$ and $b_{2}=1.015$.

Recall from Examples 3.5 and 3.11 that the optimal super replicating and riskminimizing strategies for $H=1$ depend on $\xi_{2}$ and $\lambda_{1}$, only. Thus, Figure 4.1 shows the forward rates relevant for determining the hedging strategies. Furthermore, Example 3.5 gives that the super-replicating price at time 0 corresponds to the zero coupon bond price in the conditional model given $\varepsilon_{1}=l$, which in turn corresponds to a 2-period forward rate of 0.03142 . Here, and in the remaining of the section, all numbers are given with 4 significant digits.

From Example 3.11 we furthermore note that the risk-minimizing strategy depends on $\breve{q}$. Thus, to obtain some numbers we have to specify $Q$. Henceforth, we let $\breve{p}=0.5$ and consider risk-minimization under the minimal martingale measure, i.e. $\breve{q}=\breve{p}$. The optimal super-replicating and risk-minimizing strategies and the corresponding prices are illustrated in Figure 4.2. Here, the first column gives the super-replicating price, $\hat{\vartheta}$ and $\hat{\eta}$, and the second column shows the risk-minimizing price, $\vartheta^{*}$ and $\eta^{*}$. Here, and henceforth we refer to the value of the risk-minimizing strategy as a price, since it is the arbitrage free price under the chosen equivalent martingale measure. Since the super-replicating price is an upper bound for the interval of arbitrage free prices, the price using the criterion of risk-minimization is obviously lower than or equal to the superreplicating price. In particular it is strictly lower if there is a reinvestment risk, 


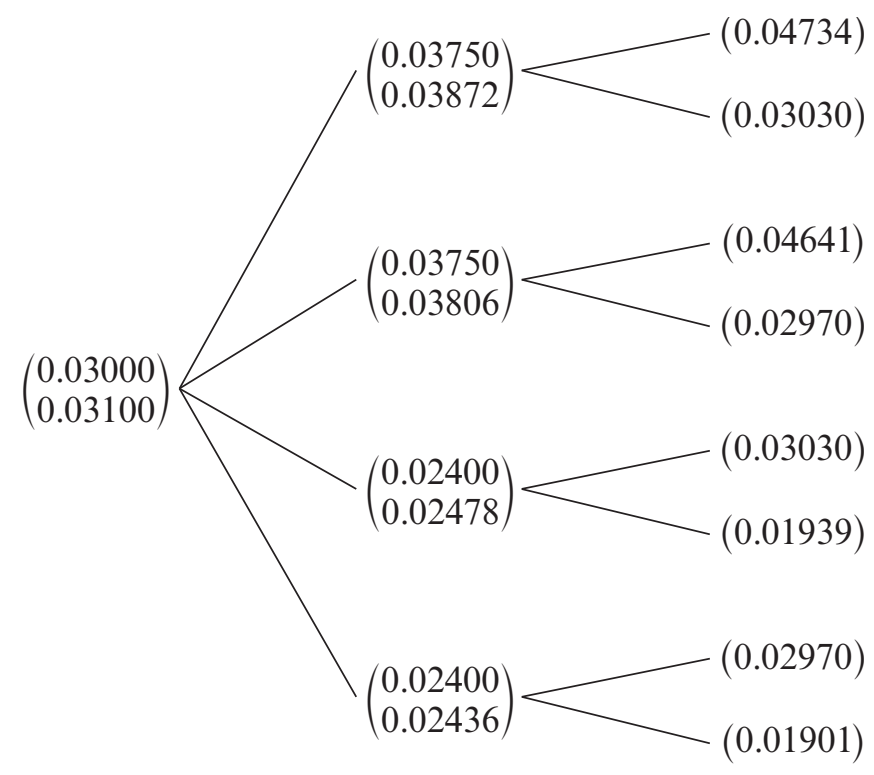

FIgURE 4.1: Relevant forward rates at time 0,1 and 2. At time 0 and 1 the vector shows the short rate and 1-period forward rate, whereas at time 2 only the short rate is relevant.

i.e. if $b_{1} \neq b_{2}$. In addition to the hedging strategies we may apply Proposition 3.7 to obtain the maximal riskfree maturity guarantee $G_{0}^{3}=1.095$ and the maximal riskfree periodic interest rate guarantee $g_{0}^{3}=0.03081$.

Now we are interested in how the prices using the criteria of super-replication and risk-minimization are affected by changing $b_{1}$ and $b_{2}$, which determine the shape of the forward rate curve at time $t, t \in\{1,2,3\}$. Investigating Table 4.1 we observe that the price at time 0 using risk-minimization is decreasing in

TABLE 4.1

A COMPARISON OF PRICES AT TIME 0 USING RISK-MINIMIZATION AND SUPER-REPLICATION. Top: Dependence on $b_{1}$. BotTom: Dependence on $b_{2}$.

\begin{tabular}{c|c|c|c}
\hline \hline$b_{1}$ & $b_{2}$ & Risk-minimization & Super-replication \\
\hline 1.05 & 1.015 & 0.9125 & 0.9130 \\
1.0325 & 1.015 & 0.9128 & 0.9130 \\
1.015 & 1.015 & 0.9130 & 0.9130 \\
\hline 1.0325 & 1.015 & 0.9128 & 0.9130 \\
1.0325 & 1 & 0.9130 & 0.9134 \\
1.0325 & 0.99 & 0.9131 & 0.9137 \\
1.0325 & 0.98 & 0.9132 & 0.9140 \\
\hline \hline
\end{tabular}




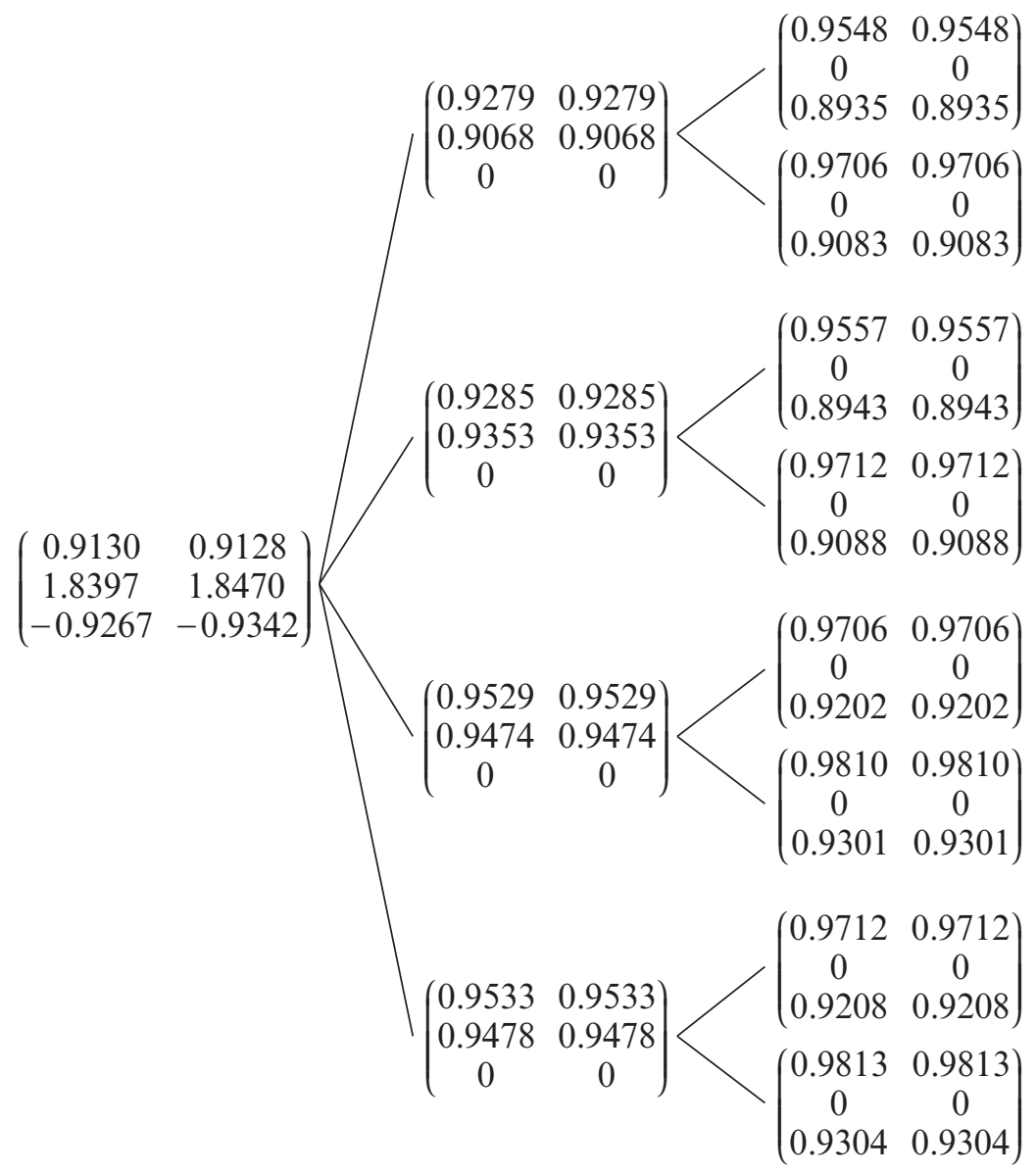

FIGURE 4.2: Hedging strategies and associated prices. First column: Super-replicating price, $\hat{\vartheta}$ and $\hat{\eta}$. Second column: Price using risk-minimization, $\vartheta^{*}$ and $\eta^{*}$.

both $b_{1}$ and $b_{2}$. This is intuitively clear since a steeper positive slope leads to lower bond prices and hence a smaller initial investment. The super-replicating price is also decreasing in $b_{2}$, however, in contrast to the risk-minimizing price, it is independent of $b_{1}$. The independence can be explained by the fact that the criterion of super-replication considers the "worst scenario" only. Furthermore, we observe that, as anticipated above, the risk-minimizing and super-replicating prices coincide when $b_{1}=b_{2}$, i.e. when there is no reinvestment risk.

\section{A comparison with practice in Danish life insurance}

The Danish life insurance companies are forced by legislation to disregard the reinvestment risk and value their long term liabilities using a yield curve, which is level beyond 30 years. Here we consider the similar principle of a level long 
term yield curve beyond the time of maturity of the longest traded bond. We shall refer to this approach as the principle of a level long term yield curve (even though we in discrete time have a yield vector rather than a yield curve). In this setting with discrete compounding the yield at time 0 of a zero coupon bond with maturity $t$ is defined by

$$
y_{0, t}=\left(\frac{1}{P(0, t)}\right)^{\frac{1}{t}}-1 \text {. }
$$

Here, the yield vector at time 0 is given by $\left(y_{0,1}, y_{0,2}\right)=(0.03000,0.03050)$. Thus, the principle of a level long term yield curve corresponds to assuming $y_{0,3}=$ $y_{0,2}=0.03050$, which leads to a price of 0.9138 . In addition to the level long term yield curve principle we introduce the analogous principle of a level long term forward rate curve, where we price using a forward rate curve, which is level beyond the time of maturity of the longest traded bond. Here, this leads to the price 0.9134 . We note that both principles only depend on the present forward rate curve, and thus they are independent of the possible future developments. Furthermore none of the principles are based on the no arbitrage principle.

We now turn to the relationship between the yield vector and the forward rate vector. When the yield vector is increasing (decreasing) the forward rate vector lies above (below) the yield vector. Thus, if we have a level long term yield vector, the long term forward rate vector is level and equal to the yield vector. On the other hand an increasing (decreasing) forward rate vector which is level for long times to maturity corresponds to a yield vector which increases (decreases) and tends towards the forward rate vector as the time to maturity increases. The increase (decrease) in the yield vector on the interval, where the forward rate vector is level, is given by

$$
y_{0, t}-y_{0, t-1}=\left(1+y_{0, t-1}\right)\left(\left(\frac{1+f_{0, t-1}}{1+y_{0, t-1}}\right)^{\frac{1}{t}}-1\right) \text {. }
$$

In this example the forward rate vector at time 0 is increasing, such that the principle of a level long term forward rate curve leads to a lower price than the level long term yield curve principle.

Now we are interested in whether the principles lead to prices in the interval of arbitrage free prices. In this simple example, where we consider a fixed claim and the time horizons $\tilde{T}=2$ and $T=3$ a principle leads to a price in the interval of arbitrage free prices if and only if the value of $f_{0,2}$ implied by the principle lies above the 2-period forward rate implied by the super-replicating price and below the 2-period forward rate implied by the "best scenario" price (which is a lower bound for the interval of arbitrage free prices). From Table 4.2 we observe that if we allow for increasing forward rate vectors only, both principles lead to a 2-period forward rate below the one implied by the super-replicating price, and hence they lead to a price higher than the 
TABLE 4.2

VALUES OF $f_{0,2}$ IMPLIED BY, RESPECTIVELY, THE “BEST SCENARIO" PRICE, THE SUPER-REPLICATING PRICE AND THE PRINCIPLES OF A LEVEL LONG TERM FORWARD RATE/YIELD CURVE. Top: Dependence on $b_{1}$. BotTom: DePEndence on $b_{2}$.

\begin{tabular}{c|c|c|c|c|c}
\hline \hline$b_{1}$ & $b_{2}$ & "Best scenario" & Super-replication & Level forward & Level yield \\
\hline 1.05 & 1.015 & 0.03250 & 0.03142 & 0.03100 & 0.03050 \\
1.0325 & 1.015 & 0.03196 & 0.03142 & 0.03100 & 0.03050 \\
1.015 & 1.015 & 0.03142 & 0.03142 & 0.03100 & 0.03050 \\
\hline 1.0325 & 1.015 & 0.03196 & 0.03142 & 0.03100 & 0.03050 \\
1.0325 & 1 & 0.03196 & 0.03096 & 0.03100 & 0.03050 \\
1.0325 & 0.99 & 0.03196 & 0.03065 & 0.03100 & 0.03050 \\
1.0325 & 0.98 & 0.03196 & 0.03034 & 0.03100 & 0.03050 \\
\hline \hline
\end{tabular}

super-replicating price. Thus, in this case both principles clearly overestimate the price. If we allow for a level or decreasing forward rate vector, the 2-period forward rate implied by the super-replicating price is lower than the one implied by a the principle of a level long term forward rate curve, and if the possible decrease is sufficiently large also lower than the one implied by using a level long term yield curve, such that the principles lead to prices, which lie in the interval of arbitrage free prices. However, especially the price obtained using a level long term yield curve is in the high end of the interval of arbitrage free prices. Note that the same information also could have been observed from Table 4.1. Based on the discussion above we conclude that the principles should not be used in situations where a decreasing forward rate curve is very unlikely. If one uses one of the principles anyhow, we recommend using the level long term forward rate principle and at the same time to keep in mind that the price (most likely) is overestimated. In situations where a decreasing forward rate vector is more likely, the principles are more likely to be accurate. The accuracy depends heavily on the situation and in particular on the correspondence between the present forward rate vector and the conditional forward rate vectors. The conclusion regarding the principles is that even though they are easy to use, their results should be used as guidelines only.

\section{ACKNOWLEDGEMENTS}

This work was carried out while the author was a Ph.D. student at the Laboratory of Actuarial Mathematics at the University of Copenhagen. Financial support from Danica Pension, Nordea Pension, Pen-Sam, PFA Pension, PKA and SEB Pension is gratefully acknowledged. Moreover, the author thanks supervisors Thomas Møller and Mogens Steffensen, as well as Rolf Poulsen for helpful comments and fruitful discussions. Comments from an anonymous referee helped improve the paper. 


\section{REFERENCES}

Aliprantis, C.D., Polyrakis, Y. A. and Tourky, R. (2002) The cheapest hedge, Journal of Mathematical Economics 37, 269-295.

BJöRк, T. (2004) Arbitrage Theory in Continuous Time, 2nd edn, Oxford University Press.

DAHL, M. (2005) A Continuous-Time Model for Reinvestment Risk in Bond Markets, Working paper, Laboratory of Actuarial Mathematics, University of Copenhagen.

El Karoui, N. and Quenez, M.C. (1995) Dynamic Programming and Pricing of Contingent Claims in an Incomplete Market, SIAM Journal on Control and Optimization 33, 29-66.

Föllmer, H. and Schied, A. (2002) Stochaistic Finance: An Introduction in Discrete Time, de Gruyter Series in Mathematics 27, Berlin.

Föllmer, H. and SchweIzer, M. (1988) Hedging by Sequential Regression: An Introduction to the Mathematics of Option Trading, ASTIN Bulletin 18, 147-160.

Harrison, J.M. and KrePS, D.M. (1979) Martingales and Arbitrage in Multiperiod Securities Markets, Journal of Economic Theory 20, 381-408.

HARrison, J.M. and Pliska, S.R. (1981) Martingales and Stochaistic Integrals in the Theory of Continuous Trading. Stochaistic Processes and their Applications 11, 215-260.

JARrow, R. (1996) Modelling Fixed Income Securities and Interest Rate Options, The McGrawHill Companies Inc.

Møller, T. (2001) Hedging Equity-Linked Life Insurance Contracts, North American Actuarial Journal 5(2), 79-95.

Musiela, M. and Rutkowski, M. (1997) Martingale Methods in Financial Modelling, SpringerVerlag, Berlin.

Neuberger, A. (1999) Hedging Long-Term Exposures with Multiple Short-Term Futures Contracts, The Review of Financial Studies 12(3), 429-459.

Rutkowski, M. (1999) Self-Financing Strategies for Sliding, Rolling-Horizon, and Consol Bonds, Mathematical Finance 9(4), 361-385.

SchweIzer, M. (1994) Risk-minimizing Hedging Strategies under Restricted Information, Mathematical Finance 4, 327-342.

SchweIzer, M. (1995) On the Minimal Martingale Measure and the Föllmer-Schweizer Decomposition, Stochastic Analysis and Applications 13, 573-599.

Sommer, D. (1997) Pricing and Hedging of Contingent Claims in Term Structure Models with Exogenous Issuing of New Bonds, European Financial Management 3(3), 269-292.

MikKel DAHL

Markets, Nordea

Christiansbro, Strandgade 3, Postbox 850,

DK-0900 Copenhagen C, Denmark

Email:mikkel.dahl@nordea.com 